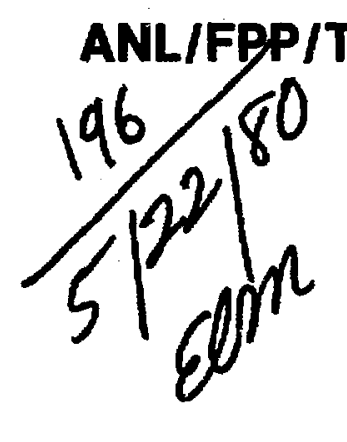

$$
\text { DR. } 1211
$$

\title{
MATERIALS PROGRAM PLAN FOR INERTIAL CONFINEMENT FUSION
} by

A. Garde, B. O. Hall, S. D. Harkness, P. S. Maiya, M. D. Rechtin, and C. -Y. LI

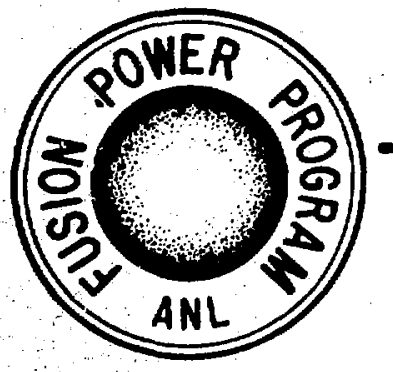

\section{FUSION POWER PROGRAM}

Argonne Mational Laberatory 9700 Seuth Cass Avenue Argonne, Minnols 60439

Prepared for the U. S. Departmont of Enery under Contract W-31-10s-Eny-38 
Distribution Categories:

Magnetic Fusion Energy (UC-20)

Reactor Materials (UC-20c)

ANL/FPP/TM-126

ARGONNE NATIONAL LABORATORY

9700 South Cass Avenue

Argonne, Illinois 60439

A. Garde, B. 0. Hall, S. D. Harkness, P. S. Maiya, M. D. Rechtin

Fusion Power Program

and

C. $-\mathrm{Y}, \mathrm{Li}$

Cornell University

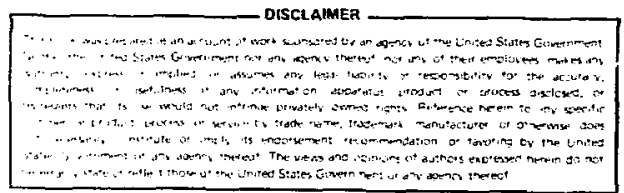

S. D. Harkness, Project Coordinator

August 1979

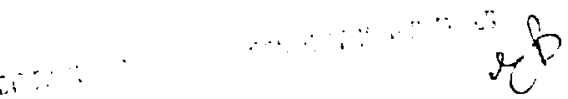


1. Introduction ................... 1

1.1 Alloy Selection. ................... 1

1.2 Environmental Parameters ........... 3

1.3 Program Plan Approach............. 4

2. Microstructural Evolution. .............. 10

2.1 ssope. ....................... 10

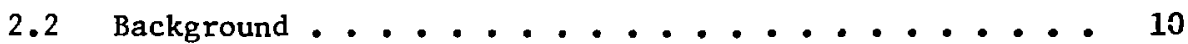

2.2.1 Steady-State Irradiation . . . . . . . 14

2.2 .2 Pulsed Irradiation ........... 15

2.3 Data Requirements and Strategy ......... 20

2.3.1 Materials Test Facility. ......... 20

2.3.2 Experimental Program ........... 24

3. Fatigue and Mechanical Properties........... 32

3.1 scope. ......................... 32

3.2 Problem Definition and Analysis......... 33

3.2.1 Mechanical and Thernal Environments. ..... 33

3.2.2 Chemical and Irradiation Environments. .... 36

3.3 Data Requirements.............. 36

3.3.1 Fatigue................... 36

3.3.2 other Mechanical Properties......... 41

3.4 strategy ...................... 42

4. Lithium Compatability and Other Environmental Effects. ... 57

4.1 scope. ....................... 57

4.2 Background ........................ 58

4.3 Data Requirements. ............. 60

4.3.1 I.thium Compatibility........... 60

4.3.2 Other Environmental Effects........ 64

4.4 Strategy .......................... 64

5. Executive Sumary................ 70 
Acknowledgment

We would like to thank the inertial confinement reactor design groups at Los Alamos Scientific Laboratory, Sandia Laboratory-Albuquerque and Lawrence Livermore Laboratory for their hospitality and their willingness to provide us with information and encouragement. 


\section{INTRODUCTION}

A materials development plan is a key ingredient in the definttion of a pathway to a commercial inertial fusion power plant. The objective of this report is to lay out a general strategy that will support the construction of both nearer term devices such as the Engineering Test Facility (ETF), and the Experimental Power Reactor (EPR) as well as the first comerclal plant. The schedule assumed for these devices is that presented in the Deutch ${ }^{1}$ report of September 1978 and is reproduced in Fig. 5-1 of the Executive Summary section.

Other major assumptions used in this planning effort include:

1. A continuing LMFBR R\&D program.

2. An expanding magnetic fusion ${ }^{2}$ materials progran, based on the recent task force planning effort.

3. A continuing Basic Energy Sciences program that will explore fundamental questions on radiation effects, lithium compatibility and mechanical properties.

4. The presence of some scheme to protect the first structural wall from short range radiation and particles with deposition times shorter than a microsecond.

The present report concentrates on the structural requirements of the reactor vessel and is done in a manner to make it as independent of driver choice as possible. Every attempt was made to find a cost-effective developmental path that concentrated on obtaining sufficient information to allow timely engineering decisions. Planning this path for an emerging technology, such as inertial confinement, must be a continually dynamic process.

Two key strategy questions were posed in the planning for each technical area. These were:

1. Is a major separate materials test facility required by this program? If so, when and at what approximate cost?

2. What are the specific areas of the required materials technology development that the inertial confinement program must consider won't be sufficiently supported by other energy technologies?

\subsection{Alloy Selection}

The EPR is expected to be the first large yolume, high plant factor test facility available for generating the statistical data on materials response 
required for engineering design. As such, it is felt to be unlikely that any alloy or alloy system that is not now already in the process of being well characterized will be used in the construction of either ETF or EPR. The approach taken in this development plan, therefore, is to assume the use of alloy systems for which a commercial industry currently exists, for which joining and fabrication technology is known, and for which there is an ongoing program to establish their response to a fast neutron environment.

Based on this premise, the suggested materials development plan concentrates on two classes of steels for application to ETF and EPR. More advanced systems, based on such elements as vanadium, niobium or titanium, would be developed through the use of the latter device as an irradiation test bed. The two classes of steels recommended to receive the most near term attention are the ferritic stainless steels as the leading candidate, and the austenitic steels as a back-up. The ferritic stainless steels are felt to be particularly appropriate for those fusion reactor designs that use some form of liquid lithium first wall protection. They may not be appropriate for designs that use magnetic deflection for protection.

Within the broad classification of ferritic stainless steels, two major categories are suggested. The first is based on a composition range of from $9-12 \% \mathrm{Cr}, 1-2 \%$ wo with small additions of other elements such as $\mathrm{V}, \mathrm{Nb}$ and $\mathrm{W}$. These steels, which are heat treated to form a tempered martensite microstructure, are expected to be stable in a lithium environment, are resistant to radiation-induced vold formation, show promise of being little affected by helium embrittlement, have strengths better or comparable to the austenitic stainless steels up to temperatures of $\sim 600^{\circ} \mathrm{C}$ and develop about half the thermal stress for a given surface heat flux than do the austenitic steels.

Additionally, they have a significantly lower blological hazard potential than the austenitic steels after decay times of 10 years or more. Commercial production of alloys in this class has centered on their application to superheater tubing in fossil power plants in Europe. Sandvik fabricates a $12 \% \mathrm{Cr}$ alloy designated HT-9 for this application while vallourec has developed a 9\%Cr steel, Em-12, for a similar purpose. Since 1974 the U. S. LFMBR program has sponsored a joint developmental effort at Combustion 
Engineering and Oak Ridge National Laboratory ${ }^{3}$ to improve the toughness, thermal stability and weldability of these alloys that has resulted in major property improvements. This class of steels has received much recent interest for application in an irradiation environment by both the LFMBR and magnetic fusion programs.

The second type of ferritic alloy is based on the $2-1 / 4 \% \mathrm{Cr}-1 \% \mathrm{Mo}$ compositional base. This steel shares many of the advantages of the $9 \% \mathrm{Cr}$ steels with respect to radiation damage resistance, lithium compatibility, and levels of thermal stresses generated under a given heat loading. It has iower creep strengths than some of the $9 \% \mathrm{Cr}$ steels and is susceptible to rusting, which is a disadvantage during field erection. The $2-1 / 4 \% \mathrm{Cr}-1 \% \mathrm{Mo}$ steels have the advantage of a strong domestic commercial experience base plus the results of an extensive research and development program supporting their selection as the reference LMFBR steam generator material.

The austenitic stainless steels as typified by Type 316 are included as back-up candidates in the event that radiation effects on the ductile-brittle transition temperature of ferritic steels result in unacceptable toughness levels at temperatures of interest. Type 316 is the most highly researched structural alloy for irradiation use, is widely available and easily fabricated and, thus, represents a fall-back position for the program.

\subsection{Environmental Parameters}

The expected flux levels, energy spectra and duration times of neutron, ion, and electromagnetic radiation that may be incident on the first surface are given in Table 1-1. The spread in these values is a result of both the assumption of different possible reactor design concepts (e.g. lithium waterfall vs. wetted wall), and the pellet design and chamber gas pressure dictated by the use of different drivers. The establishment of the assumed ranges was based on work at Livermore, ${ }^{4}$ Exxon, ${ }^{5}$ Los Alamos, ${ }^{6}$ Sandia, 7 and Wisconsin, 8 as well as on calculations conducted separately at ANL. 9,10 The interrelationship between design approaches and materials requirements is particularly important for inertial confinement development. After considering materials development requirements, one of our conclusions is that the materials effort must be closely coupled with the reactor design development effort. This appears to be an essential ingredient in finding a 
Table 1-1. Environmental Parameters

\begin{tabular}{|c|c|c|c|}
\hline & $\begin{array}{c}\text { Peak } \\
\text { Flux } \\
\left(\mathrm{cm}^{-2} \mathrm{~s}^{-i}\right)\end{array}$ & $\begin{array}{c}\text { Energy } \\
\text { Spectrum }\end{array}$ & $\begin{array}{c}\text { Deposition } \\
\text { Time }\end{array}$ \\
\hline $\begin{array}{l}\text { Neutrons } \\
\text { Ions } \\
\mathrm{X} \text {-rays }\end{array}$ & $\begin{array}{l}10^{20}-10^{21} \\
10^{18}-10^{19}\end{array}$ & $\begin{array}{l}14 \mathrm{MeV} \mathrm{G}^{\star} \\
<\mathrm{E}>=12.6 \mathrm{MeV}^{\dagger} \\
300-800 \mathrm{keV} \mathrm{M} \\
0.3-1 \mathrm{keV} \mathrm{BB}\end{array}$ & $\begin{array}{l}1-50 \text { ns } \\
1-10 \text { us } \\
10-100 \text { ps }\end{array}$ \\
\hline \multicolumn{4}{|c|}{$\begin{array}{l}\text { Spectral type } \\
\mathrm{BB}=\mathrm{Blackbody} \\
\mathrm{M}=\text { Maxwellian } \\
\mathrm{G}=\text { Gaussian } \\
\langle\mathrm{E}\rangle \text { is the average energy }\end{array}$} \\
\hline
\end{tabular}

cost-effective approach to a commercial power system based on the challenging conditions presented by inertial confinement fusion. A summary, based primarily on the work of Maniscalco 5 , of many of the relevant materials requirements resulting from the different designs is presented in Table 1-2. In this materials development planning study, some form of first wall surface protection is assumed so that the flux of ions to the first structural surface is minimized, as is the power deposited in the front surface by electromagnetic radiation.

\subsection{Program Plan Approach}

The program plan is laid out in five sections. Following the first introductory section, the second section deals with the effect of the irradiation environment on the microstructure of materials. A major part of the inftial activity in this area will be aimed toward evaluating the importance of pulse effects on microstructural development. The usefulness of existing test facilities in determining the importance of pulse effects will relate directly to the need and desirability of building a dedicated materials test facility. The approach taken in this and subsequen sections is to define the expected situation in general terms, identify the data required, point out what information is expected from other sources, and finally, present a schedule and discuss the manpower and facilities that the 
Table 1-2.

ICF Reactor Design Parameters Which Influence Material Requirements

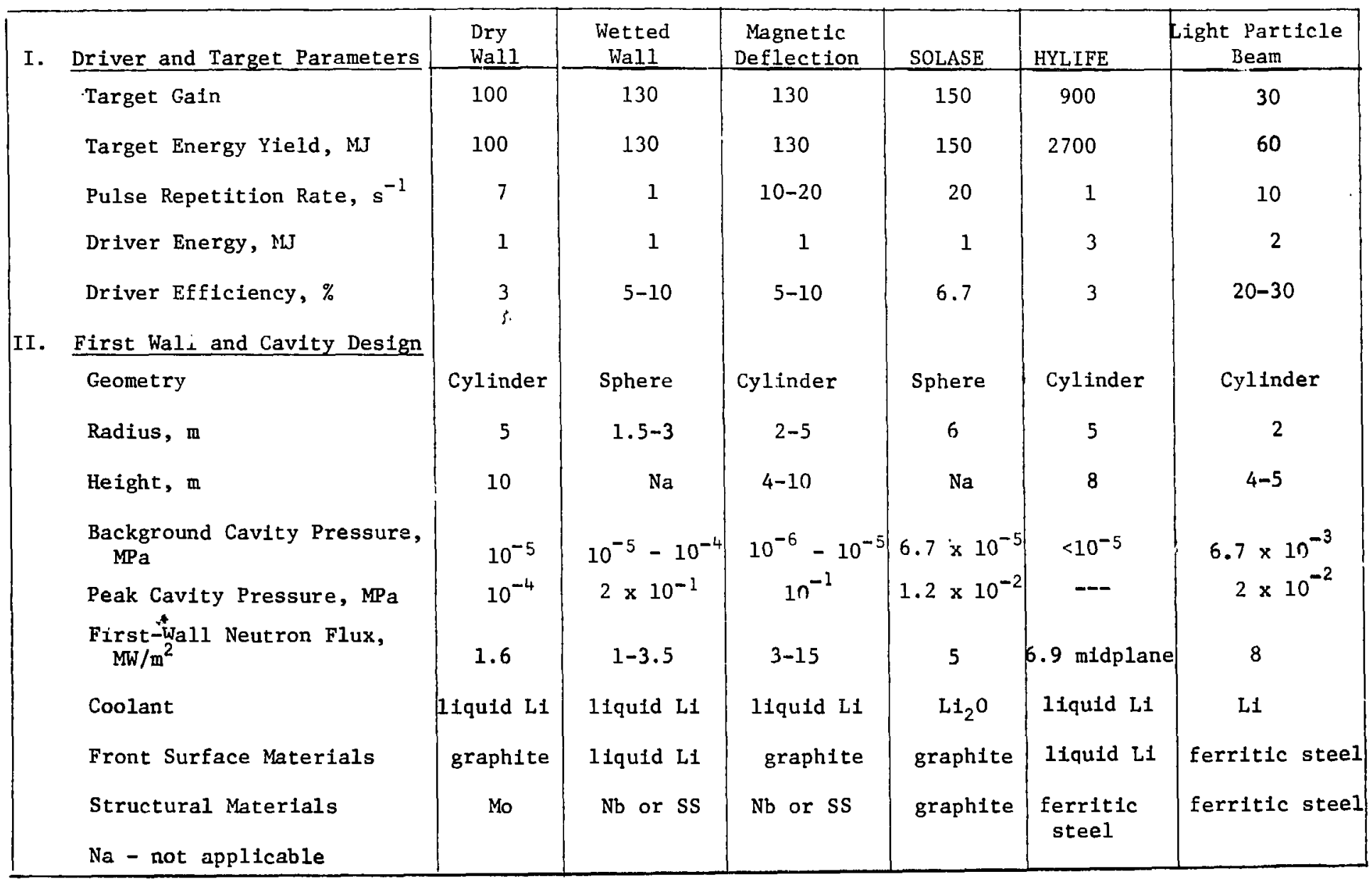


Table $\mathbf{i}-2$. (Continued)

\begin{tabular}{|c|c|c|c|c|c|c|c|}
\hline & $\begin{array}{l}\text { First Wall and Cavity Design } \\
\text { (Contirued) }\end{array}$ & $\begin{array}{l}\text { Dry } \\
\text { Wa11 } \\
\end{array}$ & $\begin{array}{c}\text { Wetted } \\
\text { Wall }\end{array}$ & $\begin{array}{c}\text { Magnetic } \\
\text { Deflection }\end{array}$ & SOLASE & HYLIFE & $\begin{array}{l}\text { Light Particle } \\
\text { Beam }\end{array}$ \\
\hline & $\begin{array}{l}\text { Desired Lifetime of } \\
\text { First-Wall, yr. }\end{array}$ & 1 & 3 & 1 & 1 & 30 & 2 \\
\hline & $\begin{array}{l}\text { Maximum Stress in Front } \\
\text { Surface, } \mathrm{MPa}\end{array}$ & 80 & $\mathrm{Na}$ & $-\infty$ & 41.4 & -- & 75 \\
\hline & $\begin{array}{l}\text { Maximum Stress in First- } \\
\text { Wall Structure, } \mathrm{MPa}\end{array}$ & 30 & $100-200$ & $100-200$ & 19.7 & 89 & 60 \\
\hline & $\begin{array}{l}\text { Maximum Front Surface } \\
\text { Temperature, }{ }^{\circ} \mathrm{K}\end{array}$ & 1800 & $\mathrm{Na}$ & $700-1270$ & 2270 & $\mathrm{Na}$ & 900 \\
\hline & $\begin{array}{l}\text { Average Front Surface } \\
\text { Temperature, }{ }^{\circ} \mathrm{K}\end{array}$ & 1200 & $570-770$ & $570-770$ & 2120 & 770 & 750 \\
\hline & $\begin{array}{l}\text { Maximum Structure } \\
\text { Temperature, }{ }^{\circ} \mathrm{K}\end{array}$ & 750 & $570-770$ & $570-770$ & 1020 & 800 & 750 \\
\hline & $\begin{array}{l}\text { Displacement Damage in } \\
\text { Front Surface, dpa/yr.* }\end{array}$ & 16 & $\mathrm{Na}$ & --- & 32.5 & $\mathrm{Na}$ & -- \\
\hline & $\begin{array}{l}\text { Displacement ramage in } \\
\text { Structure, dpa/yr.* }\end{array}$ & 11 & $10-30$ & $40-200$ & 32.5 & 2.9 midplane & -- \\
\hline & $\begin{array}{l}\text { Helium Production in } \\
\text { Front Surface, at } \mathrm{ppm} / \mathrm{yr} . *\end{array}$ & 3000 & $\mathrm{Na}$ & $4200-21,000$ & 7000 & $\mathrm{Na}$ & $\rightarrow$ \\
\hline & Structure, at ppm/yr.* & 50 & $40-300$ & $150-800$ & 7000 & 3.4 & -- \\
\hline & Coolant & 1 iquid L1. & 1iquid LI & 1iquid Li & $\mathrm{LI}_{2} \mathrm{O}$ & 11quid LI & 1iquid Li \\
\hline
\end{tabular}


Table 1-2. (Cont 1 nued)

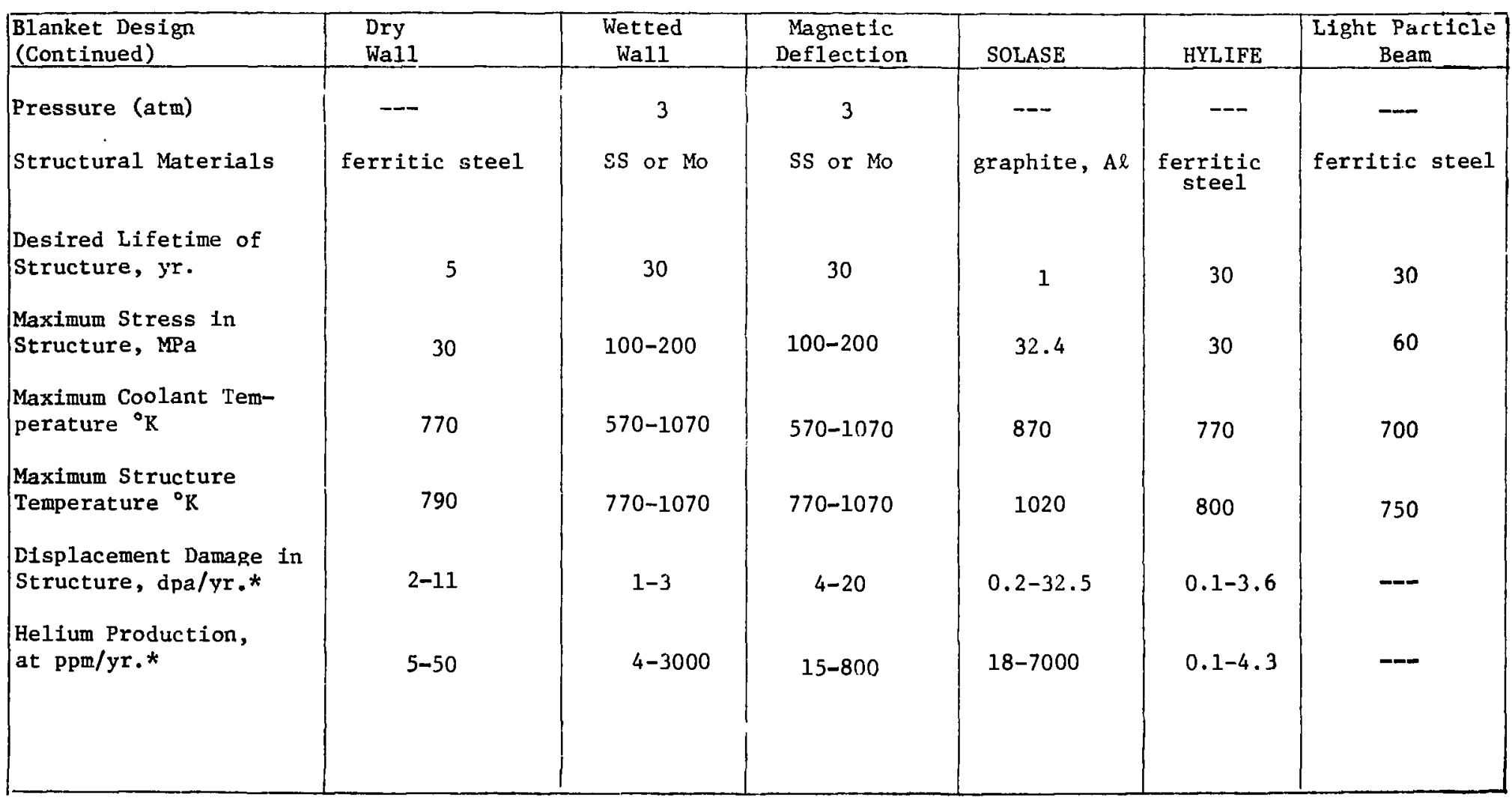

* to convert to SI units of events/s from events/yr divide by $3.156 \cdot 10^{7}$. 
Inertial fusion program must be willing to dedicate to provide the necessary information on a timely basis.

Section 3 deals with the development effort that is necessary to cope with the high cycle loading of the first wall structure. The loading pulses are expected to range from 1 to 20 per second ( $3 \times 10^{7}$ to $6 \times 10^{8} /$ year), thus creating a high cycle fatigue problem for any long-lived first wall structure. The interrelationship between specimen and component testing is a inajor issue in this section. Static mechanical property requirements are also considered here. Of particular importance are the combined effects of point defect clusters and the transmutation produced helium and hydrogen on the fracture toughness and ductility of candidate structural materials.

Lithium compatibility is treated in Section 4. It is likely that inertial confinement designs will be more dependent on the use of liquid lithium than magnetic fusion devices. This $i$ is due, on the one hand, to the fact that magnetic fields are no longer needed, thereby removing MHD effects on pumping and, on the other, on the need to provide a protective first wall layer against the extremely high $\mathrm{X}$-ray and ion power levels that result from the short time scale of the energy release process. The possible effect of pellet debris on compatibility is a subject of special consideration due to its uniqueness to inertial confinement fusion. The facility requirements for this key area must be defined early in the program.

The final section integrates the conclusions reached in the body of the report into a unified strategy that suggests a particular effort level to support major program milestones. 


\section{REFERENCES}

1. J. M. Deutch, Department of Energy Policy for Fusion Energy, DOE/ER-0018 (Sept. 1978).

2. Fusion Reactor Materials Program Plan, DOE/ET-0032 (July 1978).

3. P. Patriarca, S. D. Yarkness, J. M. Duke, and L. R. Cooper, Nuclear Technology, 28, (1976) 516.

4. J. Hovingh, "Design Considerations in Inertially-Confined Fusion Reactors", Lawrence Livermore Laboratory UCRL-78499 (Aug. 1976); W. R. Mejer and J. A. Maniscalco, "Reactor Concepts for Laser Fusion", Lawrence Livermore Laboratory, UCRL-79654 (July 1977).

5. 3. A. Maniscalco, D. H. Berwald, and W. R. Meler, "The Material Implications of Design and System Studies for Inertial Confinement Fusion Systems", First Topical Meeting on Fusion Reactor Materials, Miami Beach, FL, (Jan. 1979) to be published.

6. I. 0. Bohachevsky, "Scaling of Reactor Cavity Wall Loads and Stresses", Lo: Alamos Scientific Laboratory, LA-7014-MS (Nov. 1977).

7. D. L. Cook and M. A. Sweeney, "Critical Environmental Considerations for Particle-Beam Driven ICF Reactor Materials", Sandia Laboratories, SAND79-0222C (Jan. 1979).

8. SOLASE - A Laser Fusion Reactor Study, University of Wisconsin, UWFDM-220.

9. G. Magelssen, private communication.

10. J. M. Kramer, private communjcation. 


\section{MICROSTRUCTURAL EVOLUTION}

\subsection{Scope}

Mechanical properties of materials are strongly dependent on details of microstructure. In an inertial confinement fusion reactor (ICFR), the first wall will be subjected to displacement damage, thermal pulses, and stress pulses, all three of which can produce changes in microstructure. The purpose of this section is to discuss the features of the ICFR environment that lead to microstructural evolution in the first wall, indicate the materials problems that may arise, and outline the types of programs and facilities that are needed to assess and solve these problems. The ICFR environment is discussed first, along with the implications of various reactor designs on the approach taken in this study, and available experimental and theoretical data on the effects of the important components of the environment is reviewed. Then the data necessary to meet the ICFR programmatic goals is outiined, and a strategy for attaining the necessary information is suggested. Finally specific recommendations are made for materials testing programs based both on available testing facilities and on a possible, prospective dedicated test facility necessitated by large pulsed neutron effects or currently unforeseen testing needs.

\subsection{Background}

The materials environment of an ICFR is harsh. In an unprotected first wall, ions from the pellet debris can produce displacement damage at a rate as high as 100 displacements per atom (dpa) per second in the near surface region. X-rays can raise surface temperatures above the melting point, with thermal stress waves generated by the rapid energy deposition; the blast wave from the pellet implosion can cause pressure pulses that are capable of plastically deforming the first wall material. The instantaneous neutron dose rate following the pellet burn is orders of magnitude larger than that found in fission reactors. (See Table 2-1 for a comparison of the expected ICFR neutron environment with that in other facllities.) To compound the problem, the thermal and mechanical loadings and displacement damage are applied intermittently, providing an unique environment about which not much is currently known. 
Table 2-1 Test Facilities

\begin{tabular}{|c|c|c|c|c|}
\hline & $\begin{array}{c}\text { ICFR }^{1} \\
\text { (Li protected) } \\
\text { (14 MeV neutrons) }\end{array}$ & $\begin{array}{c}\text { RTNS }-I^{2} \\
<\mathrm{E}> \\
\sim 14 \mathrm{MeV} \text { neutrons }\end{array}$ & $\begin{array}{l}\text { ICFR }{ }^{3} \\
\text { (non } \mathrm{Li} \text { protected) } \\
\text { (14 } \mathrm{MeV} \text { neutrons) }\end{array}$ & $\begin{array}{c}\text { FMI }_{2}{ }^{4} \\
\langle\mathrm{E}\rangle^{2} \\
\sim 14 \mathrm{MeV} \text { neutrons }\end{array}$ \\
\hline $\begin{array}{l}\text { Peak Displacement } \\
\text { Rate, dpa-s }\end{array}$ & 0.1 & $3 \times 10^{-8}$ & (neutron on $1 y$ ) & $2 \times 10^{-6}$ \\
\hline $\begin{array}{l}\text { Average Displacement } \\
\text { Rate, dpa-s }\end{array}$ & $10^{-7}-10^{-6}$ & $3 \times 10^{-8}$ & $10^{-6}-2 \times 10^{-5}$ & $2 \times 10^{-6}$ \\
\hline $\begin{array}{l}\text { Peak Particle Flux, } \\
\mathrm{cm}^{-2} \mathrm{~s}^{-1}\end{array}$ & $\sim 5 \times 10^{20}$ & $10^{13}$ & $\sim 5 \times 10^{20}$ & $10^{25}$ \\
\hline $\begin{array}{l}\text { Displacement Pulse } \\
\text { Width, ns }\end{array}$ & 10 & cont inuous & 10 & continuous \\
\hline $\begin{array}{l}\text { Pulse Repetition } \\
\text { Rate, HZ }\end{array}$ & 1 & dc & $10-20$ & $\mathrm{dc}$ \\
\hline $\begin{array}{l}\text { Helium Production } \\
\text { in Structure, } \\
\text { appm-yr }\end{array}$ & $6 *-300 * *$ & 30 & $50^{\dagger}-7000^{+\dagger}$ & 3000 \\
\hline
\end{tabular}

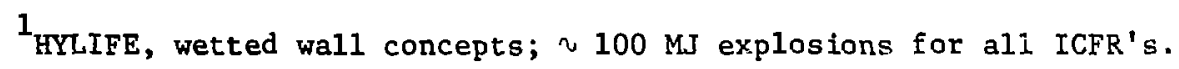

20 be avallable in 1979 for dedicated materials testing.

3 Dry metal wall, magnetic deflection, and SOLASE designs.

${ }^{4}$ To be avallable in 1982 .

*HYLIFE design.

** LASL wetted wall design.

'Dry metal wall.

${ }^{+}+$SOLASE graphite wall. 
Table 2-1. (Continued)

\begin{tabular}{|c|c|c|c|c|c|}
\hline & $\begin{array}{l}\text { Heavy Ion } \\
\text { Accelerator } \\
\text { (4 MeV nickel) }\end{array}$ & $\begin{array}{c}\text { WNR }^{5} \\
\text { (spallation } \\
\text { neutron source) } \\
(10 \mathrm{MeV} \text { avg.) }\end{array}$ & $\begin{array}{l}\text { FFTF } \\
\text { Fast Neutrons } \\
\langle\mathrm{E}\rangle \sim 0.45 \mathrm{MeV}\end{array}$ & $\begin{array}{c}\text { IPNS-I } I^{6} \\
\text { (fast neutron) } \\
E>0.1 \mathrm{MeV} \\
\langle\mathrm{E}\rangle \sim 0.7 \mathrm{MeV}\end{array}$ & $\begin{array}{l}\text { HVEM } \\
(1 \mathrm{MeV} \\
\text { electrons) }\end{array}$ \\
\hline $\begin{array}{l}\text { Peak Displacement } \\
\text { Rate, dpa-s }\end{array}$ & $0.1-0.4^{+\star}$ & $0.05-0.10$ & $10^{-7}-10^{-6}$ & $10^{-4}-10^{-3}$ & $10^{-3}$ \\
\hline $\begin{array}{l}\text { Average Displacement } \\
\text { Rate, dpa-s-I }\end{array}$ & $10^{-7}-10^{-3}$ & $10^{-7}-10^{-6}$ & $10^{-7}-10^{-6}$ & $10^{-8}-10^{-7}$ & $10^{-3}$ \\
\hline $\begin{array}{l}\text { Peak Particle Flux, } \\
\mathrm{cm}^{-2}-\mathrm{s}^{-1}\end{array}$ & $10^{12}-10^{13}$ & $2-5 \times 10^{20}$ & $10^{15}$ & $0.3 \times 10^{18}$ & $10^{15}$ \\
\hline $\begin{array}{l}\text { Displacement Pulse } \\
\text { Width, ns }\end{array}$ & 2 & 200 & continuous & 10,000 & continuous \\
\hline $\begin{array}{l}\text { Pulse Repetition } \\
\text { Rate, HZ }\end{array}$ & de to $10^{7}$ & $1-10$ & $\mathrm{dc}$ & $15-45$ & de \\
\hline $\begin{array}{l}\text { Hel Ium Product Ion } \\
\text { In Structure, } \\
\text { appm-yr-1 }\end{array}$ & $\begin{array}{l}\text { avallable with } \\
\text { dual beam in- } \\
\text { jection }\end{array}$ & $\begin{array}{l}\text { approx. same } \\
\text { as ICFR }\end{array}$ & $3-30$ & $0.3-3$ & none \\
\hline
\end{tabular}

${ }^{5}$ WNR facility to be operating with proton storage ring and able to perform as shown by 1984.

${ }^{6}$ IPNS-I to be operational by 1982 .

${ }^{\dagger *} 0.1 \mathrm{dpa} / \mathrm{s}$ corresponds to $1 \mu \mathrm{A}$ beam focused to a $2 \mathrm{~mm}$ dlameter beam of $25 \mu \mathrm{A} / \mathrm{cm}^{2}$ and $0.4 \mathrm{dpa} / \mathrm{s}$ to a $1 \mathrm{~mm}$ diameter beam with density of $100 \mu \mathrm{A} / \mathrm{cm}^{2}$. 
An initial assessment of environmental parameters and realization of the severity of the bare wall environment has led to design concepts that include some protection for the first wall. A 1ithium-wetted wall, 1ithium waterfall, magnetic deflection, or a buffer gas prevents both $\mathrm{X}$-ray and ion interactions with the wall and therefore eliminates a major portion of the surface damage. Thermal stress waves are ameliorated by the protection devices and are in the elastic regime, ${ }^{1}$ thereby generating no massive changes in the microstructure. With the exception of the lithium waterfall proposed in the HYLIFE design, the neutron flux is essentially unaffected by the protection schemes; bulk irradiation damage that is due to the neutrons thus becomes the major concern and microstructural evolution will be primarily affected by the radiation component of the environment.

\section{Neutron Effects}

The ICFR neutron environment has significant differences from that of a fission reactor. The two major difference: are its cyclic nature and the high instantaneous damage rates. Fission reactors operate in a steady-state mode with continuous neutron fluxes and damage rates of the order of $10^{-7}-10^{-6} \mathrm{dpa}-$

$\mathrm{s}^{-1}$. The ICFR will cycle at $1-10 \mathrm{~Hz}$ with a neutron pulse lasting between 1 and $100 \mathrm{~ns}$, producing displacements at rates between $10^{-2}$ and $10 \mathrm{dpa}^{-1}$. The lower damage rates are appropriate for waterfall designs, the higher rates for other protection schemes.

The interaction of neutrons with a material leads to several types of change in the material. Coupling to the electronic structure results in temperature changes, elastic collisions give displacement damage through cascade formation, and nuclear reactions produce hellum and solid transmutants. The excess point defects (vacancies and interstitials) generated by displacement events can aggregate to form dislocation loops or voids, ent ance precipitation or dissolution reactions, and cause redistribution of solute elements by enhanced diffusion or segregation. Solid transmutants can affect phase stability and harden the matrix. The products of the neutron-matrix interactions can thus cause nucleation of new microstructural features and enhanced growth or redistribution of those present before irradiation.

On the macroscopic scale, these types of microstructural changes can cause irradiation-induced deformation, i.e. swelling and creep, and loss of 
ductility. The presence of helium can lead to bubble swelling at high temperatures and grain boundary embrittlement. If segregation of solute elements to surfaces occurs, the change in surface composition may affect corrosion properties. The severity of these potential materials problems in the ICFR can be assessed if microstructural changes in the pulsed neutron environment are understood.

\subsubsection{Steady-State Irradiation}

Studies of radiation damage that were initiated to support fission reactor technology can provide some of the information necessary for understanding radiation effects on microstructure and mechanical properties. The Liquid Metal Fast Breeder Reactor (LMFBR) program has produced a large quantity of data on steady-state neutron irradiation effects in materials at constant temperature. Empirical equations that describe steady-state swelling and creep in the austenitic stainless steels have been formulated; ${ }^{2}$ ductility and stress-rupture data have been obtained from post-irradiation testing; ${ }^{3}$ and some information on precipitation effects ${ }^{4}$ and phase stability ${ }^{5}$ has been obtained. The program has focused primarily on austenitic stainless steels and the void-awelling phenomenon, with the aim of predicting its magnitıde at high doses and tailoring materials to minimize its occurrence. Because of an emphasis on design requirements and the availability of a prototypic environment in EBR-II and other fast reactors, the general approach to radiation damage in reactor structural materials has been to subject the existing data on macroscopic dimensional changes to statistical analysis and obtain empirical correlations between, for example, swelling, dose, dose rate, stress, and temperatuxe. This approach does not require a knowledge of the microstructural changes that neutrons induce, and consequently has not produced a large data base of detailed microstructural observations that are correlated with mechanical properties data.

Substantial understanding of microstructural changes caused by displacement damage has been obtained from theorctical models of individual phenomenon. The models have been used to identify important physical processes involved in microstructural evolution and to predict qualitative dependencies on irradiation parameters. The models, however, are not based on first principles and must be calibrated with detalled microstructural data from neutron irradiated materlals in order to yleld quantitative predictions. 
These data are not generally available. Ion and electron beam irradiations, 6 which can be used to simulate neutron irradiation, have provided data necessary to verify mechanisms and have been used to study, under relatively controlled conditions, the kinetics of microstructural changes. 7 However no correlation of microstructural changes under different irradiation conditions is available to link the simulation and neutron results or to extend the current data base to the ICFR environment. 8 Because ion and electron irradiations damage only the near surface region of a material, these techniques have limited use in the study of damage effects on bulk mechanical properties or of correlations between microstructures and mechanical properties.

It should be emphasized that the data available on neutron irradiation effects have been obtained under steady-state conditions, generally with flux, temperature, and stress all held constant, and typical dose rates are orders of magnitude lower than the instantaneous rates expected in an ICFR $\left(10^{-7} \mathrm{dpa}-\right.$

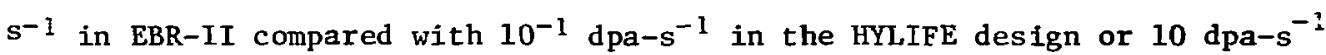
in the SOLASE design). Some experiments involving single-step changes in temperature or siress with constant flux are in progress, ${ }^{9}$ but no systematic studies have been reported. Considerations of the effects of pulsed conditions on micrustructural development have been very limited, with most of the work being theoretical using adapted steady-state computer codes. A few experiments with pulsed ion beams have been reported. This work and the results will be considered in detail later in the section.

\subsubsection{Pulsed Irradiation}

\section{Planned Magnetic Fusion Effort}

Many features of the ICFR first wall environment are also present in the magnetic fusion reactor (MFR) first-wall environment. The neutron energy spectra are similar, so generation rates for hellum and solid transmutants will be comparable. Proposed operating temperature ranges are the same for both $\left(300-500^{\circ} \mathrm{C}\right.$ for steels and $500-1000^{\circ} \mathrm{C}$ for refractories). The anticipated stress levels in ICFRs are approximately a factor of two higher than those in MFRs, due to higher instanteous heat loadings. MFRs are also operated in a pulsed mode, with $10^{3}-10^{5}$ power cycles per year for tokamak designs. In many areas, then, the materials concerns for MFR first walls must parallel those for ICFRs. 
The Task Group on Damage Analysis and Fundamental Studies (DAFS), which provides technical assistance to the Materials and Radiation Effects Branch of the Office of Fusion Energy, has prepared a program plan for magnetic fusion materials development. 10 In the area of danage microstructure evolution, the program plan emphasizes studies aimed toward understanding the interaction of helium with displacement damage, because of its anticipated effects on ductility, and development of methods to correlate damage microstructures with mechanical properties. The latter effort is necessary because of the small test volumes in available high-energy neutron facilities, and the fact that charged particle irradiations are best suited for microstructure studies. Assessments of the effects of hydrogen and solid transmutants on microstructure are also planned, along with the effects of cycling and damage rate. Most of the microstructural studies are scheduled to be completed between 1984 and 1986, and the validation and calibration of microstructural models of flow and fracture behavior during irradiation are to be finished between 1988 and 1990 .

The scope of the magnetic fusion materials program is very broad, including development of techniques to characterize various irradiation environments, stuaies of the primary damage state, measurement of high-neutron energy crosi sections for nuclear reactions and other parameters whose values are necessary for model development. Much of the information obtained from this program will benefit the ICFR materials effort.

There are two major differences between the radiation environments in MFRs and ICFRs, however, and these differences pose problems that are specific to inertial confinement devices. Current design concepts for ICFRs require power cycling at the rate of $10^{7}$ and $10^{9}$ cycles per year, which is several orders of magnitude larger than the rate expected in tokamaks. The duration of the damage pulse is $10 \mathrm{~ns}$, substantially smaller than the times anticipated for tokamaks, and the pulse amplitude is much larger. Instantaneous damage rates for tokamaks are between $10^{-7}$ and $10^{-5} \mathrm{dpa}-\mathrm{s}^{-1}$; for ICFRs between $10^{-2}$ and $10 \mathrm{dpa}^{-1}$, a very different regime. At the higher dose rates cascade interaction or overlap may change the efficiency of pointdefect production resulting in, for example, a smaller proportion of defects surviving the initial damage event and therefore fewer available to participate in microstructure changes. The impact of the duration difference can be 
partially assessed by considering the limited amount of theoretical and experimental work that has been done on pulsed damage effects.

Recent Theoretical Efforts

The work on pulsed irradiation effects has been confined to model calculations of point-defect concentrations, void nucleation rates, and void growth rates, and experimental studies of void microstructures. Processes such as creep and enhanced diffusion are expected to be proportional to the time-averaged defect concentration, and aggregation or clustering reactions proportional to the time-averaged square of the defect concentrations. An analysis of the variation of vacancy and interstitial concentrations during a single pulse can thus be used to make qualitative comparisons with steadystate behavior. Dienes ${ }^{11}$ calculated vacancy and interstitial concentrations as functions of time (or equivalently number of pulses) assuming either exponential decay to sinks or bi-molecular recombination or both as annealing modes. He found that the defect concentrations reached steadystate after a large number of cycles and compared the steady-state values with those obtained under continuous irradiation at the time-averaged damage rate. Defect concentrations averaged over the pulse cycle were generally the san : or lower than the continuous irradiation concentrations, although the mayimum was higher than the continuous irradiation result. The square of the defect concentration averaged over the pulse cycle was larger than that for continuous irradiation when only one annealing mode was considered or when the species was faster if both annealing modes were included. One infers, therefore, that processes whose rates depend on the average defect concentration will be slower during pulsed irradiation and those whose rates depend on the time-averaged square of the concentration may be faster or slower depending on tie relative mobility of the species. These corclusions are general, since they are based only on the forms of the point defect equations and therefore apply to all pulsed irradiations.

Calculations done for this study ${ }^{12}$ using parameters approprlate for 316 SS and ICFR and MFR pulse times show significant differences in the behavior of the point defect concentrations under the two sets of conditions. During the ICFR pulse cycle, the interstltial concentration rises linearly, then anneals completely; no ratcheting occurs as the number of cycles increases. The vacancy concentration rises until the end of the irradiation, drops 
slightly at the beginning of the annealing phase due to recombination with interstitials, then remains essentially constant until the beginning of the next pulse cycle. The annealing period is short compared with the vacancy lifetime, and hence ratcheting of the vacancy concentration is rapid. Under MFR conditions, on the other hand, irradiation time is long enough so that the vacancy and interstitial concentrations approach their steady-state values before the annealing phase begins, during which the concentrations of both defects drop. The differences between ICFR and MFR conditions may be more clearly illustrated if a specific phenomenon such as interstitial loop nucleation is consldered. Rate theory calculations of interstitial loop number densities indicate that nucleation is nearly complete at a dose of $210^{-4}$ dpa for a wide range of temperatures and dose rates. For a time of $10^{-8} \mathrm{~s}$ and a dose rate of $10 \mathrm{dpa-s^{-1 }}$, it is clear that under ICFR conditions this dose is not reached during a single pulse; for MFR parameters, however, the dose during a single pulse is $10^{-4}$ or larger. The average interstitial supersaturation remains low in an ICFR compared with that in an MFR. Thus an interstitial loop microstructure may develop and grow early in an MFR environment and be retarded in an ICFR. Arguments of this sort provide some insight into microstructure evolution, but many important defect reactions, such as impurity trapping, clusiering, raaiation-enhanced reaction rates, and segregation, depend on vacancy and interstitial concentrations in a complex way and can only be investigated by detailed computer calculations.

Odette and Myers ${ }^{13}$ have calculated void nucleation rates under pulsed conditions and compared the results with rates obtained for continous irradiation at the instantaneous pulse damage rate. They found, as might be expected, that nucleation rates decreased as pulse times decreased and annealing times increased. In their calculations, however, they assumed that both vacancy and interstitial concentrations varied in phase with the irradiation pulse, which is equivalent to assuming that both concentrations reach and decay from steady-state values in a time that is short compared with the irradiation and annealing times. For MFE conditions this is a reasonable assumption, especially for the interstitial concentration; for the ICFR conditions, however, this assumption does not hold, and extension of their conclusions to the ICFR pulse regime must be justified.

The nucleation and growth of interstitial loops and vacancy clusters under ICFR conditions is being calculated with a rate theory model that 
considers both defect structures simultaneously. ${ }^{12}$ preliminary results for several pulse cycles show that loop development is retarded when compared with that obtained under continuous irradiation at the time-average damage rate.

Several authors have applied vold swelling equations, which were developed to explain steady-state irradiation results, to pulsed irradiation conditions. Ghoniem and Kulcinsk ${ }^{14}$ analyzed the effect of a single pulse on the void radius. They found that void size generally decreased, except when pulsing was timed so that irradiation resumed after all vacancies produced by the previous pulse were annealed, but before voids began annealing due to thermal emission. Kmetyk, et al, ${ }^{15}$ extended Ghonfem and Kulcinski's work to include multiple pulses and temperature oscillations. Thetr calculations for Al irradiated with a pulse of $0.5 \mathrm{~ms}$ at a frequency of $120 \mathrm{~Hz}$ showed a constant void growth rate after the vacancy and interstitial concentrations reached an oscillatory steady-state. The growth rate decreased significantly from that for continous irradiation at the time-average damage rate only when a temperature excursion, due to neutron heating, was included in the dynamic equations. This work, then, as well as the other work on void nucleation and growth under pulsed irradiations suggests that void swelling may be less severe in an ICFR than in, for example, a fission reactor with the same average dpa rate. However, calculations have not been done for potential first-wall materials or for the spectfic conditions proposed in ICFR designs. No calculations have been done to analyze the behavior of phases, precipitates, or the dislocation structure under ICFR conditions.

\section{Experimental Results}

A limited amount of experimental data, obtained from ion irradiations, is available for comparison with model calculations. Taylor, et al., ${ }^{16}$ have investigated the effects of puls d $\mathrm{NI}^{+}$-ion irradiations on the void microstructure in pure $\mathrm{Ni}$. The void microstructure developed with a pulsed beam had a lower number density and a larger average void size than that which developed with a continuous beam of the time-average intensity, suggesting that pulsing reduces the vold nucleation rate and enhances the void growth rate. A series of experiments with pre-volded samples was performed to determine the effect of beam structure, that is, relative on-off times, on vold growth. A long on-short off beam structure produced the same swelling as a constant beam; very little swelling occurred for a short on-long of structure. The greatest increase in swelling occurred for equal on-off times. 
Kaletta ${ }^{17}$ has done transmission electron microscopy (TEM) analysis of the bubble population in vanadium Implanted with helium in both continuous and pulsed modes. For pulse times between 300 and $600 \mathrm{~s}$, he found that bubble swelling at low doses ( $<1 \mathrm{dpa}$ ) was increased by nearly an order of magnitude under pulsed conditions. He postulates that relaxation of small helium clusters during the annealing period increases the concentration of small helium bubbles and leads to increased bubble coalesceice in the next irradiation period. This result points to a potential problem for ICFR first walls; however, the time structure of the pulses and the irradiation conditions for these experiments corresponded to irradiation pulses long compared with the interstitial lifetime but shorter than the vacancy lifetime. Since ICFR pulse times are on the order of the interstitial lifetime, these results do not necessarily apply to ICFR conditions.

The summary given above supports two conclusions: First, different I:crimes of microstructural behavior may be defined by the different pulse times and duty cycles in MFRs and ICFRs, and second, microstructural modeling and ion bombardment are two major tools readily available for pulsed irradiation studies. They are, in fact, currently being used to assess potential problem areas.

\subsection{Data Requirements and Strategy}

In this section, the two questions posed in the Introduction are addressed, and a program for investigating the effects of pulsed irradiation investigating on microstructural evolution in the ICFR environment is outlined.

\subsubsection{Materials Test Facility}

The need for a dedicated Materials Test Facility (MTF) depends on the relative magnitude of pulsed effects on microstructural evolution. If pulsed neutrons cause on1y smal1 and understandable changes when compared with those obtained during steady-state irradiation, then the EPR can be confidently built using data obtained from steady state irradiation devices (accelerators and fission reactors). If, however, the pulsed nature of the radiation damage causes major and inexplicat 23 changes in microstructure, a pulsed materials test facility may nave to be constructed at the same time as ETF in order to supply the data to build an EPR. 
From the summary given in Section 2.2.2 it is clear that the effects of pulsed irradiation on microstructure are not well understood. More information must be obtained, especially on effects in the ferritic and austenitic stainless steels proposed for the near-term devices, before a decision on the necessity of an MTF can be made. The suggested program that allows the scoping of this problem by 1987 relies on the combined use of currently available experimental facilities and an associated theoretical modeling effort.

As can be noted in Table 2-1, several possible test beds are available.

Existing radiation test facilities include the following categories: heavy ion accelerators, ${ }^{18}$ high flux neutron sources such as the WNR facility at Los Alamos, ${ }^{19}$ or IPNS-I at Argonne, ${ }^{20}$ fast reactors such as FFTF, ${ }^{21}$ RTNS-II at Livermore, 22 the Fusion Materials Irradiation Test Facility (FMIT), 23 and high energy electron sources in the form of high voltage electron microscopes (HVEM). ${ }^{24}$ Some of these only produce microstructural damage near free surfaces (heavy ion acceleratcrs and HVEM) and some bulk damage (WNR and FFTF). In Table 2-1 ICFR irrađiation conditions are compared with a variety of radiation test facilities available now or in the near future.

The test facilities listed may be useĩul if several conditions are satisfied: (1) the simulation scheme devised must either closely approxinate expected ICFR radiation conditions or provide a damage structure which can be related by standards or modeling to an ICFR damage structure; (2) the test facilities must be readily available a large fraction of the time since a complete materials test program could extend over many years and could involve large numbers of test specimens which require well defined experimental conditions; and (3) the economics of the simulation method should not be prohibitive.

The heavy ion accelerator is capable of providing both damage rates (up

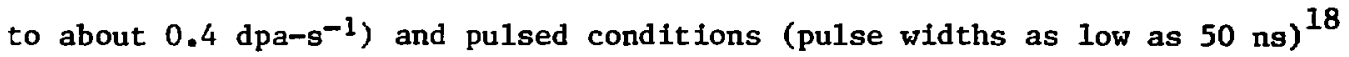
which are approximately the same as most ICFR irradiation conditions (siae Table 2-1). There are also facilities which permit simultaneous introduction of hellum gas via a second ion accelerator. ${ }^{18}$ Consequently, such an installation could be utilized to compare pulsed irradiation damage microstructures with microstructures developed during steady-state irradiations to the same total dose. With damage rates that are comparable to the midrange of those 
anticipated in an ICFR and duplication of the expected pulse cycling, ion bombardment will be best used for low dose studies, since damage will accumulate at the same rate as that expected in-reactor. The testing could be accelerated by shortening the annealing time between pulses and increasing the pulse rate from $10 \mathrm{~s}^{-1}$ to, for example, $10^{3} \mathrm{~s}^{-1}$. Care must be taken, however, to demonstrate that this acceleration does not significantly change the characteristics of the point-defect behavior or the mechanisms governing the microstructural changes. The results from low-dose ion bombardment stulies can be used to benchmark theoretical model development, and to verify whether bulk irradiation damage introduced by FFTF and/or WNR is representative of pulsed, high damage rate microstructures.

Bulk irradiation damage could be introduced by a stepwise process. The defect microstructure arising from pulsed fast neutrons could be nucleated by low-dose neutron irradiations in the WNR facility. Subsequently, the irradiation in a fission reactor such as the FFTF could be used to develop the microstructure and attain high doses. In addition, specimens may be removed from FFTF and re-irradiated in WNT. to evaluate the iterative effects of pulsed irradiations upon various representative stages of Jeveloped damage microstructures. A qualitative sampling of radiation datage trends throughout a broad range of dose and defect structures could thus be obtained, and the validity of the defect structures produced by fission reactor and/or WNR irradiations could be evaluated by comparison with those obtained in ion experiments. In this manner we inay be able to obtain an "effective" ICFR irradiation damage microstructure in specimens irradiated in FFTF and/or WNR. This step-wise irradjution would show the dominant effects of pulsed, hi.gh displacement rates upon the microstructure and provide specimens for mechanical properties tests. This method could be used to narrow the range of test materiàls and test conditions and indicate those experiments which should be performed under precise conditions for longer time periods.

Other possible radiation damage simulation facilities include high voltage electron microscopes (HVEM) and light ion accelerators. The HVEM can give a reasonably high displacement dose rate, $10^{-2} \mathrm{dpa}-\mathrm{s}^{-1}$, but the Frenkel pair damage differs substantially from the cascade damage of $14 \mathrm{MeV}$ ieutrons. Another method which may be useful is bombardment with light ions, but dose rates are substantially lower $\left(\sim 10^{-3} \mathrm{dpa}^{-1}\right.$ maximum) than desired unless the 
ion energy is extremely high (like the $800 \mathrm{MeV}$ protons at the WNR); however, the radiation damage recoil spectrum differs from that for $14 \mathrm{MeV}$ neutrons. Overa11 these alternative methods do not appear as attractive as the simulations previously discussed.

The alternative to the use of currently available irradiation simulation techniques is to prepare a dedicated materials test facility. This may prove to be a necessary step if pulsed irradiation produces unpredictable and dramatic effects on the microstructure of test specimens. A feasible approach to this procedure could be the adaptation of one of che current ICFR developmental facilities (LASL laser project, LLL laser facility, or Sandia electron beam project).

The use of a dedicated test facility is, of course, contingent upon improvement of the pellet gain. Further, the minimum repetition rate of $10 \mathrm{~s}^{-1}$ must also be attained if prototypic ICFR conditions are to be realized; this requires both high reliability of the driver system and advances in pellet injection techniques. A high plant factor will be particularly important for a dedicated facility, since its primary function would be to produce bulk damage specimens for mechanical properties tests. Ideally this requires the attainment of fluences on the order of the anticipated lifetime fluence. The minimum goal fluence should at least be that attained in a year's operation (see Table 1-1 of the Introduction). Herein lie the major drawbacks of relying solely on the concept of a dedicated test racility. It is imperative that materials testing commence by 1982 in order to provide the requisite information for the EPR design engineers. Important decisions directly concerned with materials must be made in 1986 for ETF and in 1997 for EPR. Because of the proximity of these decision dates, the most reasonable approach would be to begin materials testing with currently available accelerators, pulsed neutron sources, and fission reactors. During the course of these simulation experiments, the importance of pulsed irradiation effects should be evaluated as soon as possible, and the feasibility of the dedicated test facility idea should be re-evaluated periodically. If quantitative testing becomes impcrtant due to unpredictably large pulsed irradiation effects upon the microstructure, the dedicated test facility must be built. However, unt11 this need is demonstrated, the pressing time schedule requires that a materials testing program proceed with currently avallable irradiation testing facilities. 


\subsubsection{Experimental Program}

In accordance with the approach suggested in the Introduction, the proposed program for studying microstructural changes in the ICFR environment emphasizes the ferritic and austenitic stainless steels for near-term experimentation. Scoping studies that examine the effect of pulsed irradiation on microstructural components (e.g. precipitates, voids, dislocations) and compare microstructures obtained under pulsed and steady-state conditions are scheduled early, in order to provide information for the decision on a dedicated materials test facility. Low-dose experiments using ion accelerators are suggested for initial efforts, because of their immediate availability. Studies of damage rate and pulse duration effects on microstructures may be most important because of the potential for accelerated testing either by decreasing annealing times or increasing the damage rate. The utility of data on pulsed effects obtained in the magnetic fusion energy (MFE) program can also be evaluated with the information gained.

The study of synergistic effects between displacement damage and helium, hydrogen and transmutant effects, and the preparation of mechanical properties test specimens by WNR and/or FFTF irradiations are scheduled later in the program, while the MFE program is completing studies in these areas ${ }^{10}$ so that full benefit may be derived from their effort.

Large scale testing of advanced alloys should be deferred until the EPR is available as the major test facility.

The suggested experimental program must be supported by a theoretical modeling effort. Much of this is of a fundamental level appropriate for Division of Basic Energy Sciences (BES) support, and is relevant to understanding the effects of both magnetic and inertial fusion irradiation environments. For example, both experimental and theoretical evidence shows that helium can strongly affect microstructural evolution; a knowledge of its properties and behavior in candidate materials is therefore essential. Specific items of information that are important include its production rate and diffusion properties; Its interaction with other microstructural components such as dislocations, grain boundaries, cavities, dispersed phases, and specific to ICFR concerns, the dense cascade structure present at high damage rates; and its redistribution under cyclic temperature and stress conditions. Hydrogen production, diffusion, and distribution may be important along with its chemical 
behavior. Data on high-neutron energy cross sections should be obtained. Production rates and migration characteristics of solid transmutants, their precipitation or redistribution behavior under cyclic conditions, and their influence on other elements of the microstructure are needed. Vacancy and interstitial production rates and production efficiencies as a function of damage rate (equivalently cascade density), migration under pulsed conditions, interactions with helium, hydrogen, and impurities are some of the point defect properties that need to be measured. Characterizing the primary damage state and dense cascade structure and development at high damage rates will be important.

Once the basic processes and parameters are understood, they can be incorporated into existing models for void and loop nucleation and growth, solute segregation, helium bubble formation and growth, precipitate coarsening, etc. These models are limited in scope, but can be used to estimate magnitudes of changes in individual components of the microstructure. Surface energies, defect and solute migration energies, defect binding energies, elastic energies associated with extended structures such as precipitates, coarsening and phase transformation kinetics, and thermodynamic properties of the candidate alloy systems are included in these physical models as parameters. In some cases the development of a microstructural feature depends strong1y on a parameter: for example, void nucleation rate on surface energy or solute segregation on interstitial solute binding energy; it will then be important to have accurate values. The validity of currently available models under ICFR conditions will have to be determined; this is an area of concern because the neutron pulse times are short compared with the time scales of many physical processes. Unless it becomes clear that basic assumptions of the theories may be invalid under ICFR conditions, the major development of physical models can be left to BES and MFE programs. In order to study interactions among different microstructural elements, many investigators are developing comprehensive models that include several phenomena. An example is the inclusion of mobile helium in void nucleation and swelling theories, another is the simultaneous treatment of interstitial loop and void nucleation and growth. To obtain maximum benefit from the analytical work, experimental measurements of microstructural changes in well-characterized environments that are in some controlled way representative of the ICFR environment need to be compared with theoretical predictions. The microstructural data required include dislocation densities and structures, precipitate size distributions, 
interstitial loop densities and size distributions, gas distributions, bubble and void sizes and number densities.

If the development of microstructural elements under controlled conditions is understood, comprehensive modeling can be used to assess interactions between the different elements and to extend the available data base to ICFR conditions. With a correlation between microstructure and mechanical properties, an assessment of first-wall response can be made.

Tables 2-2, 2-3, and $2-4$ contain schedules and manpower requirements for Investigations aimed at determining the magnitude of pulsed neutron effects on microstructure, evaluating the interaction of helium and displacement damage in a pulsed irradiation environment, and studying the relationship of microstructure and mechanical properties. The focus of the last effort is on mechanisms - testing, for example, theoretical and model predictions of inreactor creep rates or confirming the predicted dependence of mechanical properties on various elements of microstructure. Programs for accumulating design data are discussed in the section on fatigue and mechanical properties. 


\section{Table 2-2.}

Material: Ferritic and Austenitic Stainless Steels

Objective: To determine the magnitude of pulsed neutron effects on microstructure and compare microstructures obtained under steady-state and pulsed lon bombardment conditions.

Scope: The effects of damage rate, dose, and pulse duration on the development of key microstructural components will be investigated.

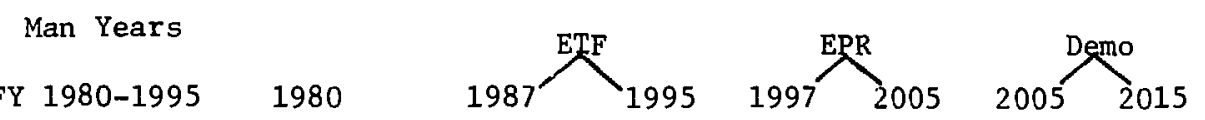

A. Specimen selection

B. Test matrix development
(a) damage rate effects
(b) dose effects
(c) pulse duration effects

C. Irradiations

D. TEM examinations

E. Analysis and modeling
40
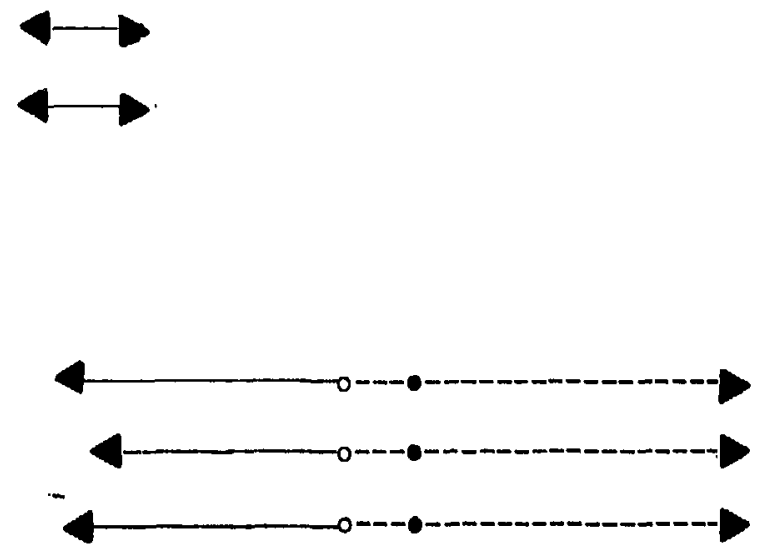

- Decision date on Materials Test Facility

- Anticipated MTF operation, if MTF warranted. 
Table 2-3.

Material: Ferritic and Austenitic Stainless Steels

Objective: To evaluate synergistic effects of helium and displacement damage under ICFR pulsed conditions using dual-beam ion bombardment.

Scope: The effects of simultaneously produced helium key microstructural components will be investigated.

\section{Man Years}

FY $1980-1995$

1980
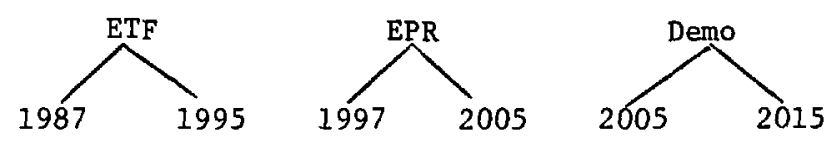

$\stackrel{N}{\infty}$

A. Test matrix development

20

(a) He/dpa ratio effects

(b) dose effects

B. Irradiations

C. TEM examination

D. Analysis and modeling

Winput from MFE helium effects program expected 
Table 2-4.

Material: Ferritic and Austenitic Stainless Steels

Obfective: To obtain mechanical properties data from specimens irradiated in WNR and/or FFTF.

Scope: Bulk damage specimens will be prepared by irradiations in WNR and FFTF with validity of microstructures evaluated using lon bombardment data; mechanical properties data will be obtained.

\section{Man Years}

FY $1980-1995$

1980
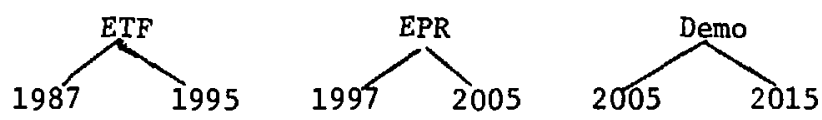
A. Irradiation technique development
B. Validation of micro- structures TEM examination
C. Irradiations - sample preparation
D. Post-irradiation testing
E. Microstructure and mechanical properties correlation

40
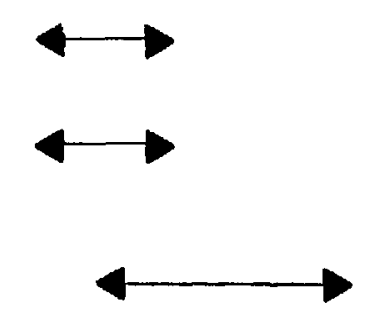


\section{REFERENCES}

1. I. O. Bohachevsky, L. A. Booth, T. G. Frank, and J. H. Pendergrass, "Loads and Stresses in ICF Reactors," 5th Inter. Conf. on Structural Mechanics in Reactor Technology, Berlin, Germany (Aug. 1979) LA-UR 79-432.

2. Nuclear Systems Materials Handbook, Vo1. 1, Design Data, TID-26666.

3. See Proc. Inter. Conf. on Radiation Effects in Breeder Reactor Materials, M. L. Bleiberg and J. W. Bennett, Eds., Scottsdale, AZ (1977).

4. D. I. Potter and H. A. Hoff, "Irradiation Effects on Precipitation in $\gamma / \gamma$ ' Ni-Al Alloys," Acta Met., 24 (1976) 1155.

5. P. Wilkes, "Phase Stability Under Irradiation - A Review of Theory and Experiment," University of Wisconsin, UWFDM-271 (Nov. 1978).

6. D. I. R. Norris, "The Use of the High Voltage Electron Microscope to Stimulate Fast Neutron-Induced Void Swelling in Metals," Jour. Nuc1. Mater., 40 (1971) 66.

7. See Proc. of the Inter. Conf. On Fundamental Aspects of Radiation Damage in Metals, M. T. Robinson and F. W. Young, Eds., Gatlinburg, TN, CONF-751006 (1975).

8. See Proc. Workshop on Correlation of Neutron and Charged-particle Damage, CONF-760673(1976).

9. J. P. Foster and A. Boltax, "Effects of Temperature Changes on $20 \%$ Cold Worked AISI 316 Stainless Steel Swelling," Westinghouse Advanced Reactors Division, WARD-OX-3045-45 (Feb. 1979).

10. The Fusion Reactor Materials Program Plan, DOE/ET-0032 (July 1978).

11. G. J. Dienes, "Pulsed Irradiation: Annealing and Accumulation of Defects," Rad. Effects, 36 (1978) 101.

12. B. 0. Hall, unpublished.

13. G. R. Odette and R. Myers, "Void Nucleation during Pulsed Irradiation," preprint.

14. N. Ghoniem and G. L. Kulcinski, "Void Growth Kinetics Under an Irradiation Pulse," University of Wisconsin, UWFDM-138 (Oct. 1975).

15. L. N. Kmetyk, W. F. Sommer, J. Weertman, and W. V. Green, "An Analytic Comparison of the Effect of Steady State and Cyclic Pulsed Radiation on Void Growth and Swelling," First Topical Meeting on Fusion Reactor Materials, Miami Beach, FL (Jan. 1979).

16. A. Taylor, D. I. Potter, and H. Wiedersich, "The Effects of Pulsed Irradiation on Void Microstructures in Nickel," to be published in Jour. Nuc1. Mater. 
17. D. Kaletta, "The Microstructure of Vanadium Implanted with 2-MeV Helium Ions under Pulsed Conditions," First Topical Meeting on Fusion Reactor Materials, Miami Beach, FL (Jan. 1979).

18. A. Taylor, J. R. Wallace, D. I. Potter, D. G. Ryding, and B. Okray Hal1, "Argonne National Laboratory Dual-ion-irradiation System," Proc. Inter. Conf. on Radiation Effects and Tritium Technology for Fusion Reactors, CONF-750989, VoI. I, (1976) I-158.

19. D. M. Parkin, "On the Feasibility of a Neutron Radiation Effects Facility at the WNR", Los Alamos Scientific Laboratory.

20. T. Blewitt, ANL, private communication.

21. User's Guide for the Irradiation of Experiments in the FTR, HEDL-MG-22 (July 1975)

22. P. J. Persiani, "A Technical Critique of Radiation Test Facilities for the CTR Surface and Materials Program", ANL/CTR 75-1.

23. Proc. of Inter. Conf. on Radiation Test Facilities for the CTR Surface and Materials Program, ANL/CTR 75-4, Argonne National Laboratory (July 1975).

24. R. L. Lyles, ANL, private communication. 


\section{FATIGUE AND MECHANICAL PROPERTIES}

\subsection{Scope}

Fatigue is likely to be the predominant failure mode in all the proposed inertial confinement fusion (ICF) systems because of the pulsed nature of operation. The present program plan is intended to meet the design need based on fatigue and other mechanical criteria. It is recognized that the degradation of allowable material fatigue properties in an ICF environment occurs by corrosion, erosion and mass transfer, irradiation damage, helium and hydrogen effects. In particular, emphasis is made to insure that the design and operation requirements of EPR are adequately fulfilled.

At present, the proposed designs are still in the conceptual stage. The details of the component configuration and design are not yet established. However, as mentioned earlier, the choice of the structural materials for near-term ICF systems can be confined to the ferritic and austenitic stainless steels. This allows the definition and formulation of a laboratory test program with test conditions based on the information available from the various ongoing design efforts. In order to confirm the adequacy of the mechanical design, the present program includes component testing, which is expected to be accomplished by means of simulation experiments, and also experiments to be performed in ETF. It should be emphasized that the proposed program is not restrictive but is flexible enough to allow timely incorporation of new design considerations and experimental results into the test matrix.

Tensile, load relaxation, creep, creep-to-rupture and fracture toughness data on the structural materials are also required for the first wall structural design. For many of the ferritic steels and austenitic stainless steels of interest, these basic mechanical properties are available from LMFBR and MFR programs. ${ }^{1,2}$ Additional work is probably not needed except in the area of fracture toughness. Ferritic steels are known to suffer from temper embrittlement due to thermal aging, and also from the embrittling effects of irradiation, and helium, hydrogen and lithium exposure. The extent of embrittlement resulting from these causes in an ICFR can be different from that in an MFR and should be properly evaluated. 


\subsection{Problem Definition and Analysis}

In this section, the mechanical, thermal, chemical and irradiation environments of the ICF systems to which the structural materials are exposed will be examined to form a basis for defining the design data requirements.

\subsubsection{Mechanical and Thermal Environments}

Recently, several investigators ${ }^{3-7}$ have addressed the sources of loadings for some ICF design concepts and have made approximate estimates concerning the magnitude of the stresses. Their results will be reviewed as a part of the background.

Fuel pellet microexplosion and blanket thermal expansion generate various mechanical loads on the first wall structure. In addition, spatial temperature gradients inside the first wall structure generate thermal loads. As mentioned earlier, the proposed program assumes that the first wall will be protected by various schemes, e.g., wetted wall, lithium fall or gas protected first wal1. The sources of load will consequently be different depending on the first wall protection scheme. In the following sections, the sources and approximate magnitudes of the mechanical and thermal loads will be considered separately.

\subsubsection{Mechanical Loads}

For the wetted wall type of design, Bohachevsky and co-workers ${ }^{3,4}$ have estimated the magnitude of various mechanical loads. The conversion of debris kinetic energy into pressure on the inner first wall surface is a source of mechanical load for background $g$ ss densities below $10^{13}-10^{14}$ atoms $/ \mathrm{cm}^{3}$. A typical value for this type of load is a fraction of an atmosphere. When the ambient density in the cavity exceeds $10^{13}-10^{14}$ atoms $/ \mathrm{cm}^{3}$, the pellet explosion w11l generate a blast wave. For a total fuel pellet energy yield of $500 \mathrm{MJ}$ with $20 \%$ of the total yield retained in the debris, the blast wave will produce a pressure of approximately $20 \mathrm{~atm}$ on the wall assuming a cavity radius of $5 \mathrm{~m}$. A most significant source of load is that produced by ablationevaporation recoll. The ablation and evaporation of lithium will occur 
following the absorption of X-ray pulse and plasma debris. The latter effect will be less important because the pulse duration for the debris is much longer than that for the X-rays. For a total pellet yield of $500 \mathrm{MJ}$ with $5 \%$ of the total yield in the form of $1 \mu$ second pulse of $\mathrm{X}$-rays, the estimated pressure from ablation is approximately $60 \mathrm{~atm}$ at a $5 \mathrm{~m}$ radius for a 1ithium-wetted wall. For the wetted-wall design, the thermal expansion of blanket material (1ithium) will also exert a pressure on the channel walls following the deposition of the neutron-energy in the blanket. It is reasonable to assume that some type of channel structure will be required for lithium low as a part of the first wa11. The propagation and reflection of pressure waves will also produce loads on the channel walls. Bohachevsky and co-workers have estimated the lithium pressure produced by thermal expansior: for the case where lithium is contained in two concentric spherical shells. For the inner shell, the calculated pressure is roughly 10 atm and is compressive.

With the estimated mechanical loads for the appropriate first-wall design, the static stress in the first wall can be calculated. For example, for a thin spherical shell, the membrane stress in the shell is RP/2S where $S$ is the thickness, $P$ is the internal pressure, and $R$ is the cavity radius. Based on the values of load given previously, the membrane stress in the thin spherical shell will in general be below the yield stress. Bohachevsky and co-workers ${ }^{3,4}$ and other investigators ${ }^{5}$ have pointed out that since the pressure pulse duration is extremely short $\left(10^{-4}-10^{-5} \mathrm{~s}\right)$ the stress in the first vall should be determined from an analysis of dynamic response. These investigators have performed calculations for a thin spherical shell subjected to an internal pressure pulse. Their results suggest that the amplitude of the stress wave is a fraction of the static values.

For the first wall protected by a lithium fall, the mechanical loads discussed earlier will not be applicable. Glenn and Young ${ }^{5}$ have estimated the pressure exerted on the first wall produced by the disassembly of an annular 1ithium fall. The pressure pulse consists of a strong pulse of approximately $150 \mathrm{MPa}$ of a few us duration which is followed by a weak pulse with a duration of approximately $100 \mu \mathrm{s}$. For a cylindrical reactor of a radius of $2-6 \mathrm{~m}$, the 
maximum hoop stress (as calculated) ${ }^{5}$ is $\sim 60 \mathrm{MPa}$ which occurs for approximately $800 \mu \mathrm{s}$ after the initial impact. The stress in the first wall protected by a lithium fall will therefore also be below the yield stress of the wall material.

\subsubsection{Therma1 Loads}

Static thermal loading will be produced by the steady state temperature gradient in the first wall structure. At a mean temperature of about $500^{\circ} \mathrm{C}$ for the first wall, it is anticipated that the resulting thermal stress will relax as a function of time. The magnitude of the thermal stress and its rate of relaxation have been examined. ${ }^{8}$ It can be assumed that with proper design, static thermal loading will not produce thermal stresses exceeding the yield stress.

Thermal stress pulses can be produced by the rapid application of thermal loads. When the energy of pellet explosion is deposited near a solid surface at a rate exceeding the rate at which heat can be conducted away into the interior, one would expect the generation of thermal stress waves. The magnitudes of this type of stress wave have been calculated. ${ }^{3,4,6}$ For the interest of the proposed program, the presence of the lithium layer at the wetted wall will protect the solid surface so that the thermal stress waves may not be an important consideration.

For an experimental reactor, the frequency of start-up and shut-down will be higher than that anticipated in a commerical plant. During the start-up and shut-down, transient thermal stresses can be significantly larger than the stresses discussed earlier. If the structural materia: is embrittled by irradiation, helium, hydrogen or temper embrittlement, one can expect structural failure produced by a brittle fracture mode. In the proposed program, the measurement of fracture toughness will be included. It is important that the fracture toughness of the first wall materials be properly evaluated as a function of the anticlpated service history so that a useful basis for defining the mechanical design limits can be developed.

It is important to point out that the above considerations are valid for a simple solid wall and do not include complexities that arise from the pregence of brazes, welds, joints and composite structures present in real 
components. Complexities may also arise from the need for using optical ports and other problems associated with driver systems. Consideration should also be given to the transient thermal stresses associated with start-up/ shut-down cycles, which are expected to be more frequent in EPR than in demonstration plants. Therefore, in the definition of data requirements, an allowance is made to insure that adequate information will be obtained so that it is aplicable to a range of anticipated situations.

\subsubsection{Chemical and Irradiation Environments}

The composition and $m_{i} i c r o s t r u c t u r e$ of the first wall structural materials are expected to be influenced by the chemical and irradiation environments present in the ICF systems, which will lead to significant degradation of the mechanical properties. The embrittlement of structural materials by neutron irradiation has been investigated in detail. However, the fatigue behavior as influenced by neutron irradiation as well as corrosion effects associated with 1 ithium exposure are not well established. The effects of lithium exposure on structural materials may involve several mechanisms. It can simply be a surface effect which leads to corrosion fatigue or it can alter the bulk composition and microstructure which may result in the deteriration of fatigue resistance. In the proposed program, the above effects will be investigated in a systematic manner. The characterization of the appropriate chemical and neutron irradiation environments have been described elsewhere in this report.

\subsection{Data Requirements}

\subsubsection{Fatigue}

Two types of fatigue design data are required in the Materials Development Plan. The first type is cbtained from laboratory experiments conducted on test specimens with simple geometries. The second type of data is generated by using actual or simulated components to characterize synergistic effects of various environments as well as to test the validity of design practice. The following section describes the details of the data requirements. 


\subsubsection{Laboratory Test Data}

Since the first wall structure of an ICFR will be subjected to cyclic loading and will experience cycles $>10^{8}$ during its life-time, there exists a potential for damage resulting from high cycle fatigue. Therefore, experiments and analyses are required to demonstrate that the structural materials will be strong with respect to fatigue loadings in the appropriate environment and that fatigue crack propagation will not lead to premature failure of the first wall.

Laboratory test data will be needed for a wide range of structural materials that will be characterized by fatigue behavior, fatigue limits, crack initiation and crack propagation behavior as influenced by surface damage and environments encountered in different ICF conceptual designs. These studies should be undertaken to include the effects of several variables (e.g., temperature, cyclic frequency, neutron irradiation (both steady and pulsed) and surrounding environment (lithium) and helium and hydrogen effects). In addition, the effects of a number of metallurgical variables (e.g., heatto-heat variations, thermal aging, grain size, etc.) on fatigue life and crack initiation and crack propagation behavior should be determined.

In the program prior to the operation of EPR, the materials of interest are ferritic steels and austenitic stainless steels. For a SOLASE-type concept, some fatigue tests will be performed on graphite. In the post-EPR program, it is anticipated that a wide range of materials currently being considered in the alloy development program will be incorporated.

The cyclic properties of the first wall materials in the laboratory can be presented in the following form:

$$
\begin{aligned}
& \text { (i) } \mathrm{S}-\mathrm{N}_{\mathrm{f}} \text { curves }\left(\mathrm{S}=\text { stress, } \mathrm{N}_{\mathrm{f}}=\right.\text { cycles to failure) } \\
& \text { (ii) } \mathrm{S}-\mathrm{N}_{\mathrm{o}} / \mathrm{N}_{\mathrm{f}} \text { curves }\left(\mathrm{N}_{\mathrm{o}} / \mathrm{N}_{\mathrm{f}}=\right.\text { fraction of life spent in crack initiation) } \\
& \text { (iii) } \mathrm{da} / \mathrm{dN}-\Delta \mathrm{K} \text { curves }(\mathrm{da} / \mathrm{dN}=\text { crack growth rate and } \mathrm{K}=\text { stress intensity). }
\end{aligned}
$$

The thermal fatigue properties may be represented in the form of $\Delta \mathrm{T}-\mathrm{N}_{0}$ curves for the various geometries and materials where $\Delta T$ is the temperature range of cycling and $\mathrm{N}_{0}$ is the crack initiation life defined in a specific manner. 
Fatigue 1ife can be established under a varlety of loadings (in a load- or strain-control mode) that are of interest to ICF reactors, and linear-elastic fracture mechanics techniques can be used to study crack propagation behavior. Investigations concerned with fatigue limtts of laboratory specimens should address fatigue behavior based on a distinction between crack initiation and crack propagation, especially if the fatigue life is dictated by crack-initiation considerations as in the case of high-cycle fatigue. Even under low-cycle fatigue conditions, the per cent of life spent in initiating a crack length of about $0.1 \mathrm{~mm}$ in smooth laboratory specimens can be as large as $50-90 \%$ depending upon the strain range of cycling ${ }^{9}$ but this can decrease significantly if surface roughness is introduced. 10 It should be noted that the real components of the ICF systems may not be smooth and in addition the first wall materials might experience erosion and pitting by contact with liquid lithium or lithium impact. Inasmuch as high-cycle fatigue behavior is extremely sensitive to surface roughness ${ }^{11}$ and surface-integrity, the effects of surface roughness and other forms of surface damage (introduced either during machining or under in-service conditions) would be an important consideration in the design of the first wall, if it is to last as long as 30 years. It is also likely that both the chemical (lithium) and neutron irradiation environments affect the high-cysie fatigue life through the effects on crack initiation behavior. For example, neutron irradiation may impart resistance to high-cycle fatigue failure predominantly by delaying the initiation of cracks even though the hardening and embrittlement associated with neutron irradiation are expected to increase the crack-propagation rate. It is also important to point out that the fatigue properties derived from isothermal loading may not be directly applicable to thermal fatigue. One of the possible ways of investigating the thermal fatigue resistance of materials is by the "Glenny disk" method. 12 The ranges of the important test variables are:

Temperature: In general, fatigue life decreases and fatigue crack growth rates increase with increasing temperature. The magnitudes of these temperature effects should be quantified in the temperature range from 550$900^{\circ} \mathrm{K}$.

Cyclic Frequency: In the several ICF conceptual designs, under normal operating conditions, it is expected that the frequency of cycling will be 
$1-20 \mathrm{cycles} / \mathrm{s}$. Superimposed upon the main cycle of operation, there could occur a number of secondary waves which may lead to additional detrimental effects. For a commercial fusion reactor, the cyclic frequency can vary from the above high frequency range (normal operating conditions) to low frequencies (those associated with the reactor start-up/shut-down cycles). Some of the transient thermal stresses could lead to brittle fracture. In a lithium environment which is corrosive, cyclic frequency can have a significant effect upon fatigue life and crack initiation and crack propagation behavior. It is, therefore, important to characterize the effect of frequency of loadings in the appropriate environment applicable to various ICF reactor concepts. In addition, the loading waveform is a mixed spectrum of severai frequencies and therefore should be an important consideration.

Stress Rat 1o (Mean-Stress Effects): The first-wall structural materials in the several ICF design concepts will experlence loadings having a nonzero stress ratio, which can have a significant effect upon fatigue life and crack propagation rates especially if the stress ratio is greater than -1 (tensile mean stress). During its life-time, the first wall will experience loadings from different events representing many stress ratios and it is important that the effect of mean stress be known and a method be available for including the effects of mean stress for use in structural analysis.

Surface Damage: As discussed previously, surface damage is an important consideration in high-cycle fatigue and therefore experiments on specimens with controlled surface damage are needed. Surface damage can be introduced in a controlled manner by low-energy ion sputtering, lithium contact, or impact machining and grinding. Composition and microstructural changes that may occur in the surface region assoctated with the introduction of surface damage should be investigated.

Multiaxiality: The actual vessels are either spherical or cylindrical, and the state of stress is multiaxial. It is recommended that some biaxial fatigue tests be performed.

Helium Effects: The effects of different helium contents and its distribution, (produced by either tritium decay or thermal neutron irradiation), on high cycle fatigue life and fatigue crack propagation will be investigated. 
Hydrogen Effects: The effects of hydrogen on high-cycle fatigue life and crack propagation will be investigated by performing the appropriate tests at vartous hydrogen pressures.

Lithium Effects: Two types of experiments will be performed to evaluate the effects of lithium on cyclic properties: post-exposure and in-situ experinents. In both types of experiments, the impurity content in lithium will be controlled to simulate the ICF conditions.

Irradiation Effects: The irradiation effects will be investigated mainly through the postirradiation tests conducted on specimens with microstructures (typical of those expected in ICF environments) produced by different techniques outlined elsewhere in the report. Wilh the availability of ETF and EPR facilities, in-situ experiments will be performed as a part of the proposed Materials Development Plan.

\subsubsection{Component Testing}

It is well known that the dominant mode of failure in a modern aircraft is high-cycle fatigue and that the su cess of the large commercial airliner as an engineering system is heavily, pendent on component testing as well as laboratory specimen testing. There ore, component testing should be an important part of the Materials Der \& spment and Testing Program Plan. The component testing can be performed on several components at various levels ranging from small parts to full : zale structures and can include synergistic effects of mechanical loads and miterials variables such as weldments. This is a necessary proof test for the design practice to ensure high reliability of the system.

The first wall of an ICFR will be a composite structure including channels and supports required for lithlum flow and for the varfous protection schemes. With the anticipated complexity, the first wall can be envisioned to be equivalent to the wing structure of an aircraft. Therefore, it will be difficult to develop and construct an EPR which is expected to have service life longer than 10 years without taking advantage of the engineering practice of the aircraft industry.

It is not straightforward to simulate the ICF environment and thermal loads in the laboratory. However, stress analysis and other relevant design calculations can be performed to estimate the magnitude and the frequency 
of the various mechanical loads. Therefore, an important part of the proposed program plan will be the development of laboratory capability to simulate the required mechanical loads and to perform the appropriate tests on the various sections of the first wall in a systematic manner. The duration of some of these tests can be as long as several years. With the availability of an ETF, component testing can be conducted in a realistic environment. It is believed that a proper combination of laboratory specimen tests and component testing will ensure the successful design and operation of an EPR.

\subsubsection{Other Mechanical Properties}

The important sources of stress in the first wall structure, as discussed in the previous section, are static thermal loads produced by the steadystate temperature gradient and transient thermal loads produced by reactor start-up and shut-down and other temperature fluctuations. These thermal loads can be relaxed by inelastic deformation in the structural material. The rate of load relaxation, which in an ICFR will be influenced by irradiation-enhanced inelastic deformation (creep), is required in order to estimate the variation of stress level as a function of time. Tensile data are required so that the design stress can be specified below the yield stress. For thin section components under a stable load, the creep-to-rupture data are used to define design limits.

Tensile data are required for a temperature range of from room temperature to $873^{\circ} \mathrm{K}$, a strain rate range of $10^{-3}$ to $10^{-6} \mathrm{sec}^{-1}$. and for both irradiated and unirradiated specimens. The required creep and load relaxation data will be for a temperature range 573 to $873^{\circ} \mathrm{K}$, for a strain rate range of $10^{-3}$ to $10^{-9} \mathrm{sec}^{-1}$, and also for irradiated and unirradiated specimens. The creep-to-rupture data required will be for the same temperature range with a range of testing times up to $10^{4}$ hours and for Irradiated and unirradiated specimens.

The fracture toughness should be evaluated by charpy impact testing and by fracture mechanics methods. The temperature range of interest is from below room temperature to $873^{\circ} \mathrm{K}$ with specimens embrittled by thermal aging, irradiation, helium, hydrogen, and lithium exposure. 


\subsection{Strategy}

From the viewpoint of reducing cost and $t$ ime, it is necessary to take advantage of other available sources of test data, and laboratory and component testing capabilities. Test data will be generated by the LMFBR and magnetic fusion and energy sciences programs. These data may not be directly applicable to the ICF systems but they can be used to expand the data base to be generated in the proposed program so that improved capabilities for the correlation and extrapolation of the data can be developed. The laboratory testing capabilities developed in the LMFBR programs for fatigue testing in sodium and neutron irradiation environments as well as component-testing capabilities developed in the aircraft industry are a few examples of established technology which can be fully utilized in the present program.

The required design data on basic mechanical properties of ferritic steels and austenitic stainless steels are expected to be available from LMFBR and MFR programs. Some post-irradiation tests and in-reactor load relaxation and creep tests may be required if the irradiated microstructures produced in ICFRs are proven to be significantly different from those produced, for example, in MFRs. The heat treatment conditions for ferritic steels for ICFR application may not be the same as those for MFRs or LMFBRs and may lead to changes in the basic mechanical properties. Some mechanical testing on unirradiated materials will therefore be required. When evaluating these mechanical properties, advantage should be taken of recent developments in the characterization of inelastic deformation in order to save effort and to facilitate data correlation and extrapolation. ${ }^{13}$

The fracture toughness of a material is known to be sensitive to temperature and to the sectional thickness of the specimen. Presently available methods based on fracture mechanics considerations are not yet adequate for predicting, from laboratory data, the fracture toughness of a material the sectional thickness of which is different from that of the laboratory specimen. It is important in the laboratory work, therefore, to use specimens of thickness equivalent to that of the components of the first wall structure whenever possible. The dimensions of irradiated specimens and lithium exposed specimens may be limited by experimental space considerations, and the development of correlations for fracture toughness evaluation as a function of sectional thickness will be important. 
Basic mechanical properties for graphite are also required. It is expected that much of the design data will be availabe from MFR programs.

The development of the Materials Program Plan will provide timely input into DOE supported design efforts. As the design effort progresses, various alternate design concepts will evolve and these changes could have a significant impact on the test conditions and the requirements of the proposed plan. One of the important considerations in the present plan is to keep close contact with the design efforts so that the relevant information concerning the design changes can be incorporated in the test program. In addition, important findings originating from the other efforts of the present plan, such as compatibility in liquid lithium and neutron irradiation studies, will be appropriately incorporated in the proposed program and used to adjust priorities. For a given material, an extensive data base will be generated in air and inert gas environments. From this data base, selected tests will be conducted in different environments to establish the effect of that environment on fatigue limits and fatigue crack initiation and propagation behavior. This approach will result in subtantial savings of time and cost. 
Table 3-1.

Material: Ferritic Steel and Austenitic Stainless Steel

Environment: Air and Inert Gas

Objective: To evaluate the fatigue and mechanical design data requirements for ETF and EPR.

Scope: The effects of a large number of important variables on fatigue limit, fatigue crack initiation and crack propagation in air and inert gas environments will be examined.

(a) Specimen testing and
development
(b) Temperature
ETF temperature
Other temperatures
(c) Frequency and waveform
ETF frequency and
waveform
Other frequencies and
waveforms


Table 3-1. (Continued)

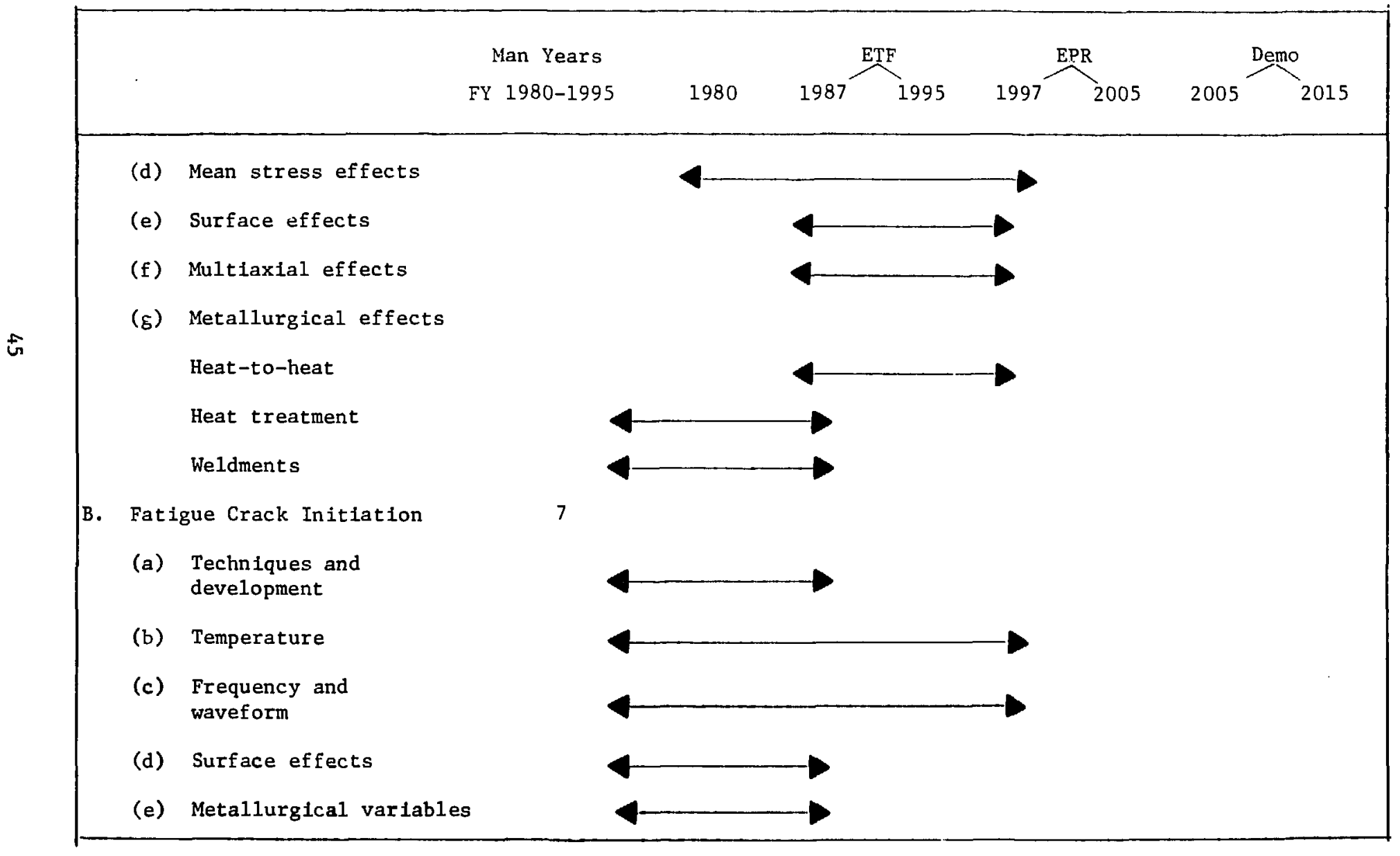


Table 3-1. (Continued)

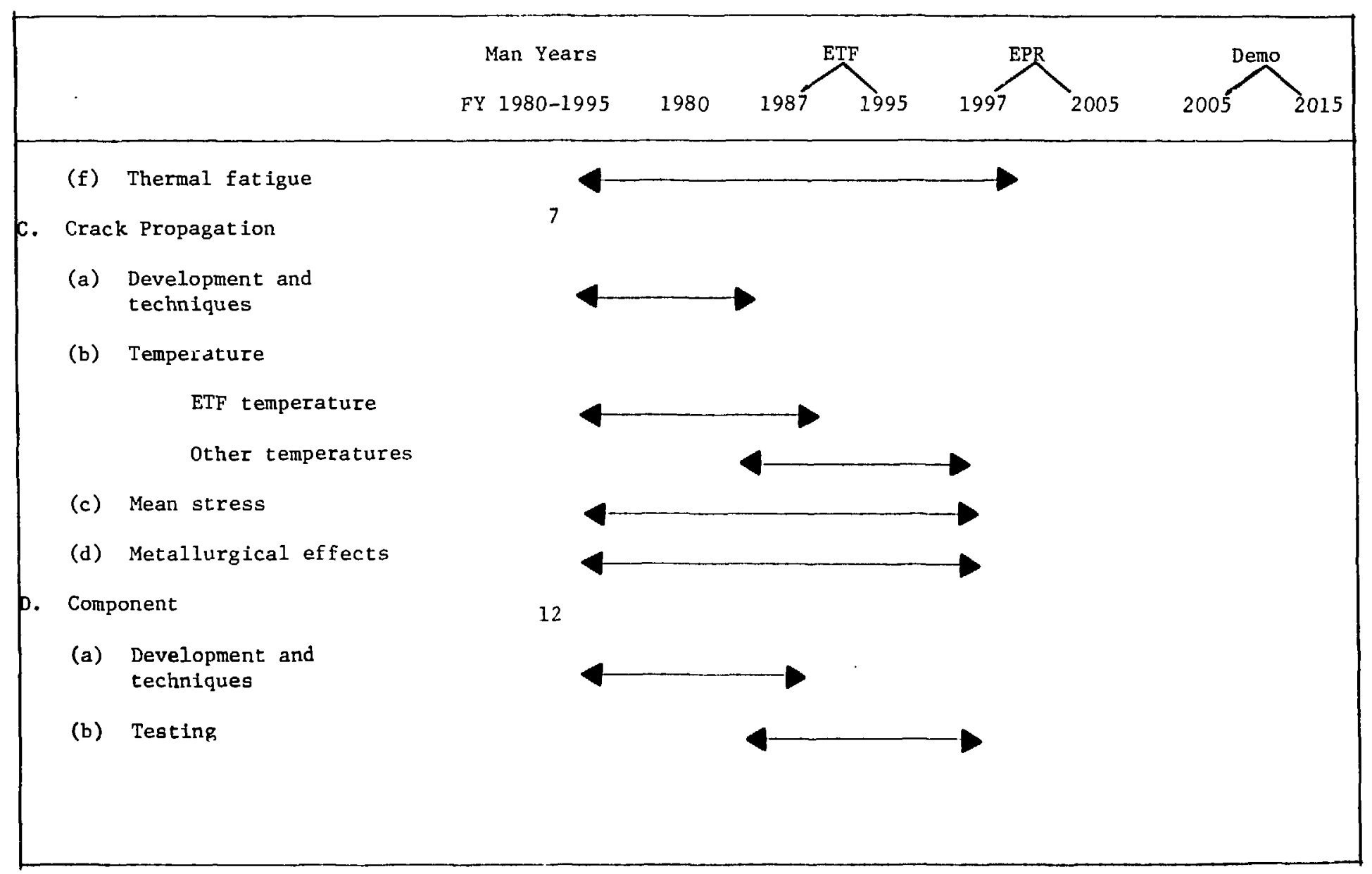


Table 3-2.

Material: Ferritic Steel and Austenitic Stainless Steel

Environment: Lithium

Objective: To evaluate the fatigue and mechanical design data requirements for ETF and EPR.

Scope: The effects of a large number of important variables on fatigue limit, fatigue crack initiation and crack propagation in lithium will be investigated.

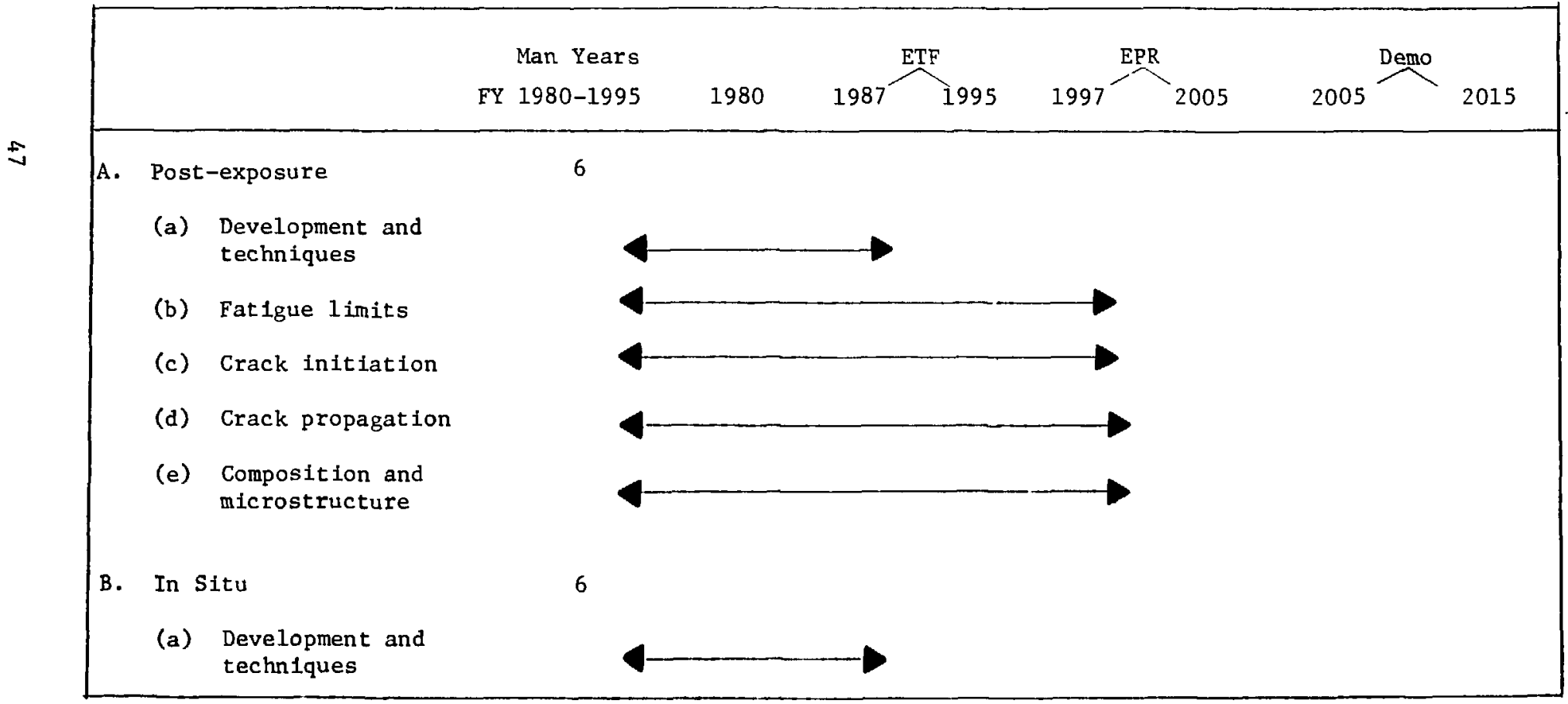


Table 3-2, (Continued)

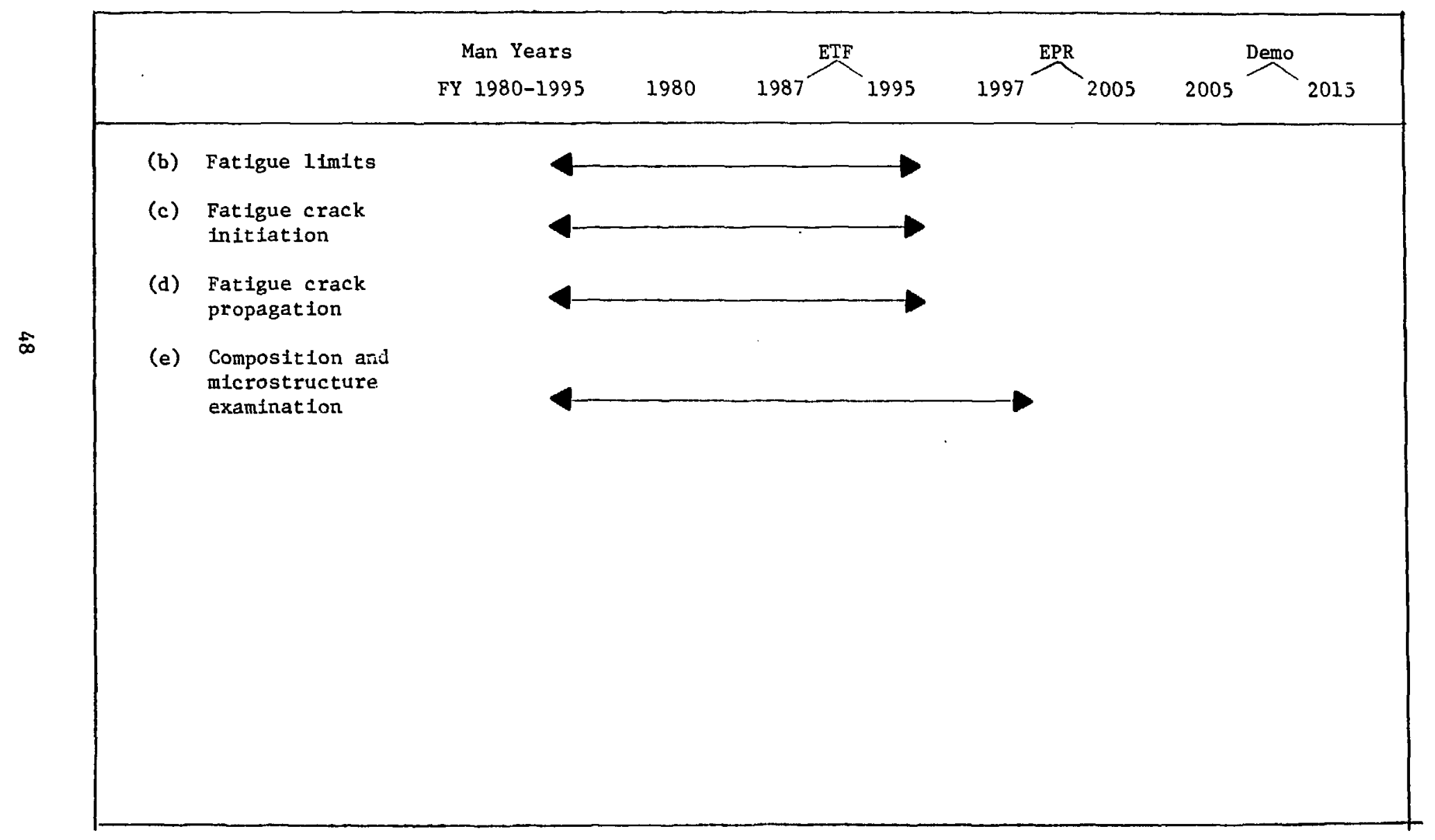


Material: $\quad$ Ferritic Steel and Austenitic Stalnless Steel Containing Helium

Environment: Inert Gas

Objective: To evaluate the fatigue and mechanical design data requirements for ETF and EPR.

Scope: The effects of helium content and its distribution on fatigue limit, fatigue crack initiaticin and crack propagation in an inert gas environment will be examined.

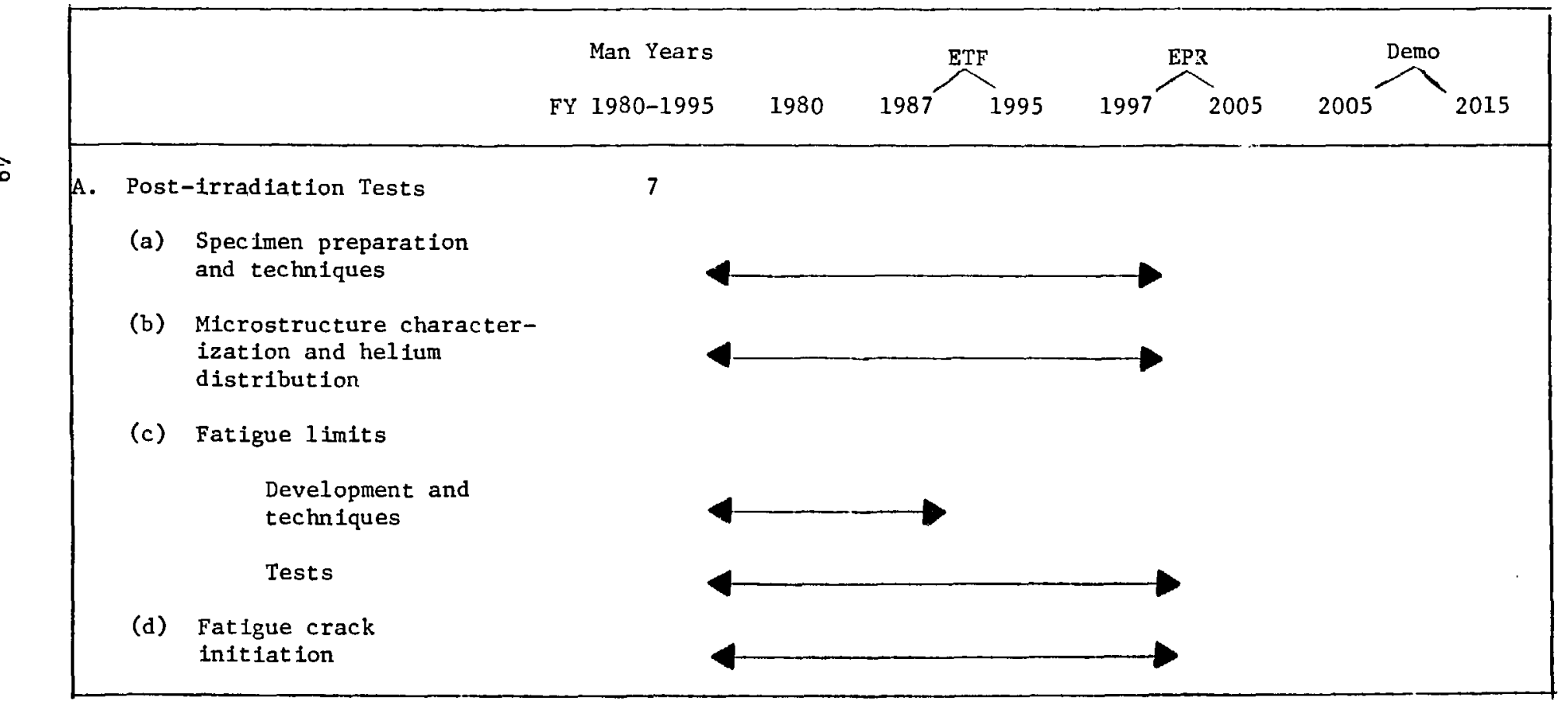


Table 3-3. (Continued)

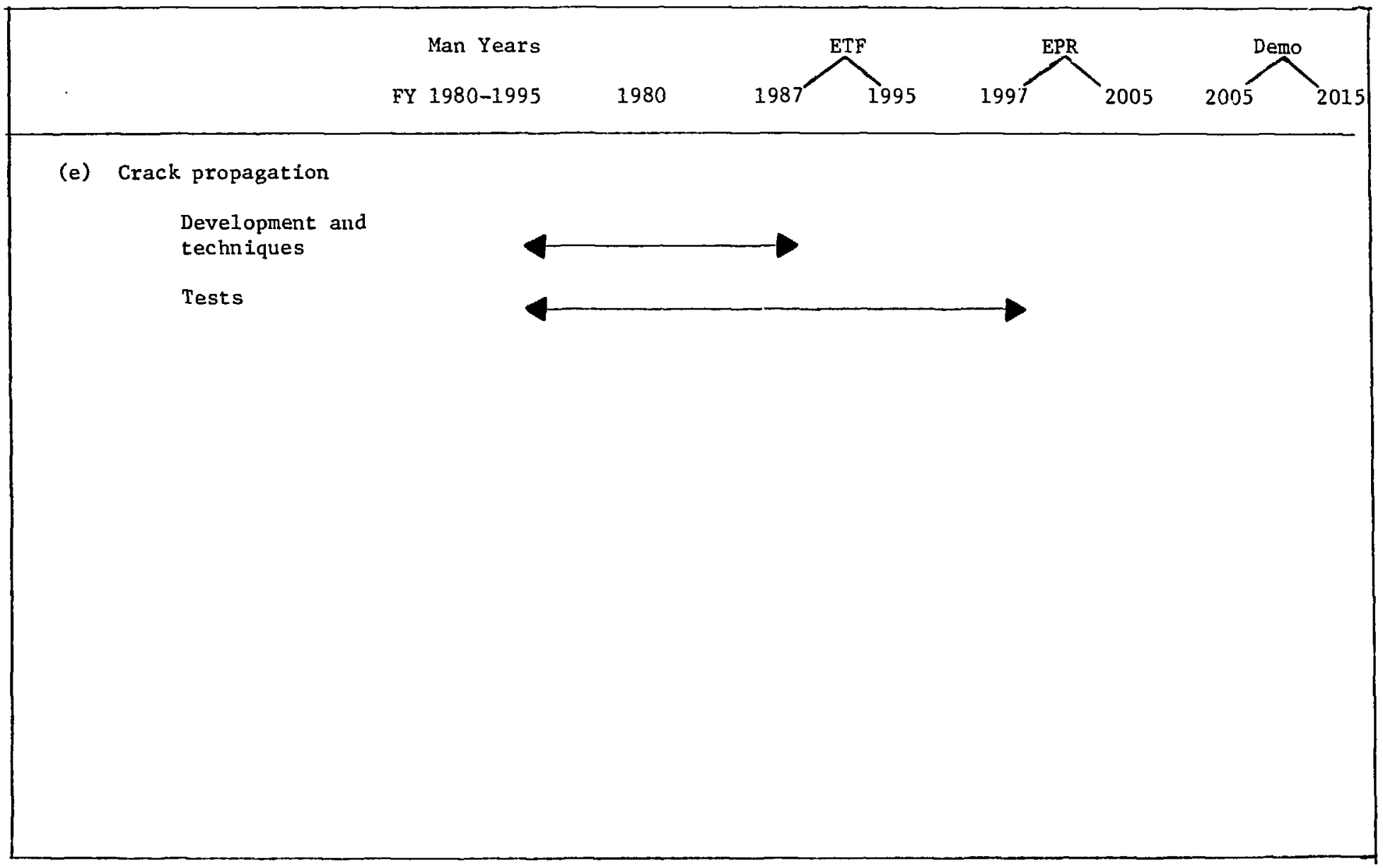


Table 3-4.

Material: Ferritic Steels anỏ Austenitic Stainless Steel

Environment: Hydrogen

Objective: To evaluate the fatigue and mechanical design data requirements for ETF and EPR.

Scope: The effects of hydrogen on fatigue limit, fatigue crack initiation and crack propagation in hydrogen will be investigated.

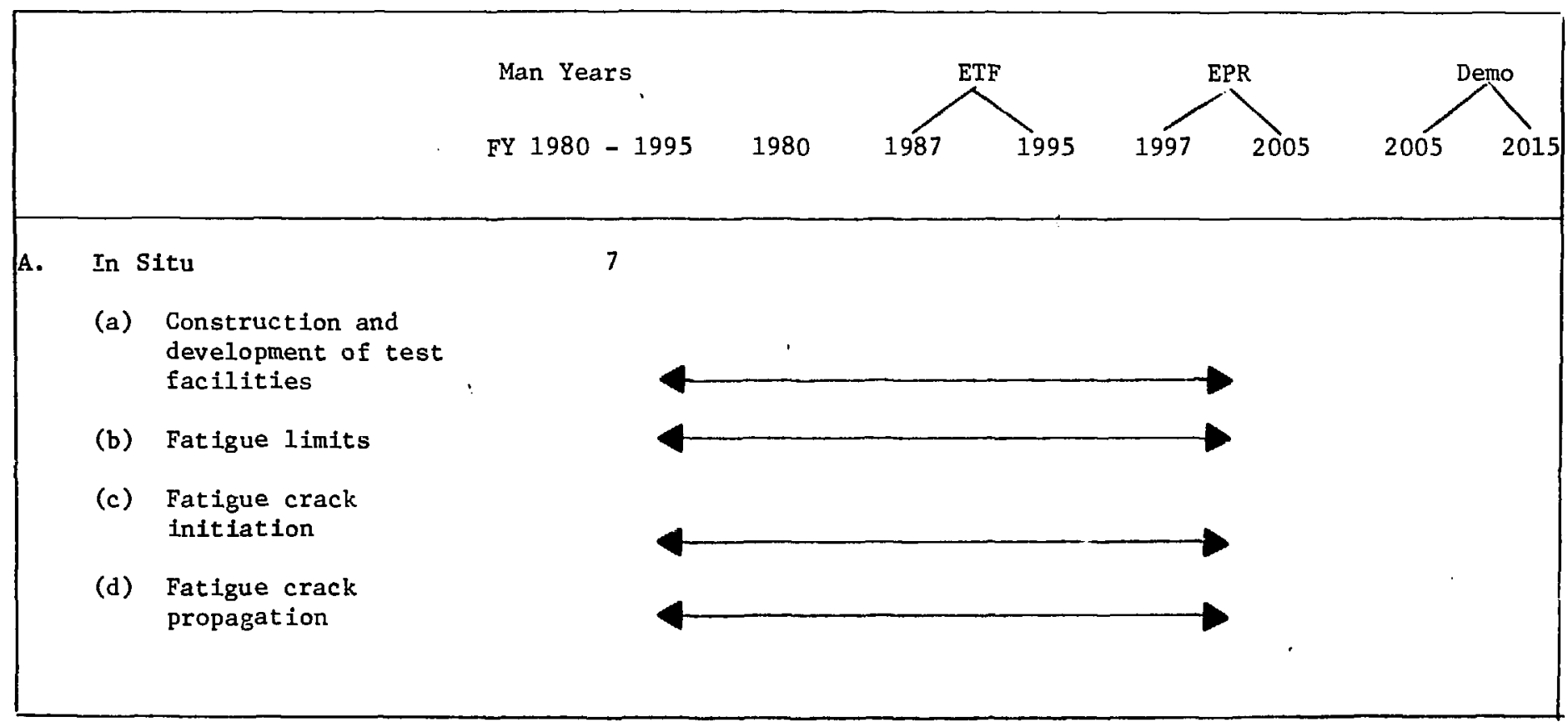


Table 3-5.

Material: Ferritic Steels and Austenitic Stainless Steel

Environment: Neutron Irradiation

objective: To evaluate the fatigue and mechanical design data requirerents for ETF and EPR.

Scope: The effects of neutron irradiation on fatigue limits and fatigue crack propagation will be investigated by both post-irradiation and in situ experiments.

A. Post-irradiation
(a) Develop techniques
(b) Fatigue limits
(c) Fatigue crack
Bropagation
In Situ ficilities
(a) Develop techniques
(b) Fatigue crack
propagation


Table 3-6.

Material: Graphite

Environment: Inert Gas

Objective: To evaluate the fatigue and mechanical design data requirements for ETF and EPR.

Scope: The effects of a large number of variables on fatigue limit in an inert gas environment will be investigated.

Man Years

FY $1980-1995$

1980
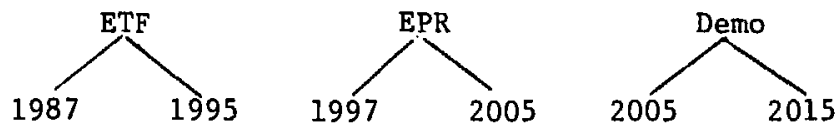

A. Fatigue Limits

(a) Specimen testing and development

(b) Temperature

(c) Frequency and waveform

(d) Mean stress effects
3

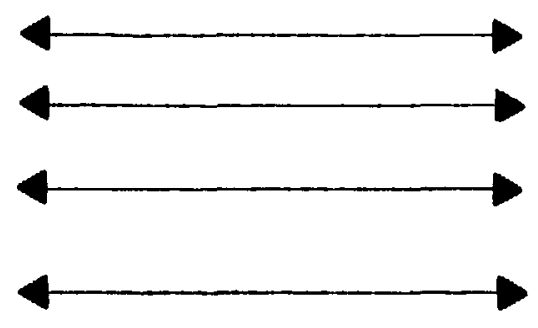


Table 3-7.

Materials: Advanced Alloys

Environments: A1r, Inert Gas, Lithium and Neutron Irradiation

Objective: To evaluate the fatigue and mechanical design data requirements after EPR.

Scope: The effects of a large number of variables including hydrogen and helium on fatigue limits, fatigue crack inftiation and crack propagation will be investigated.

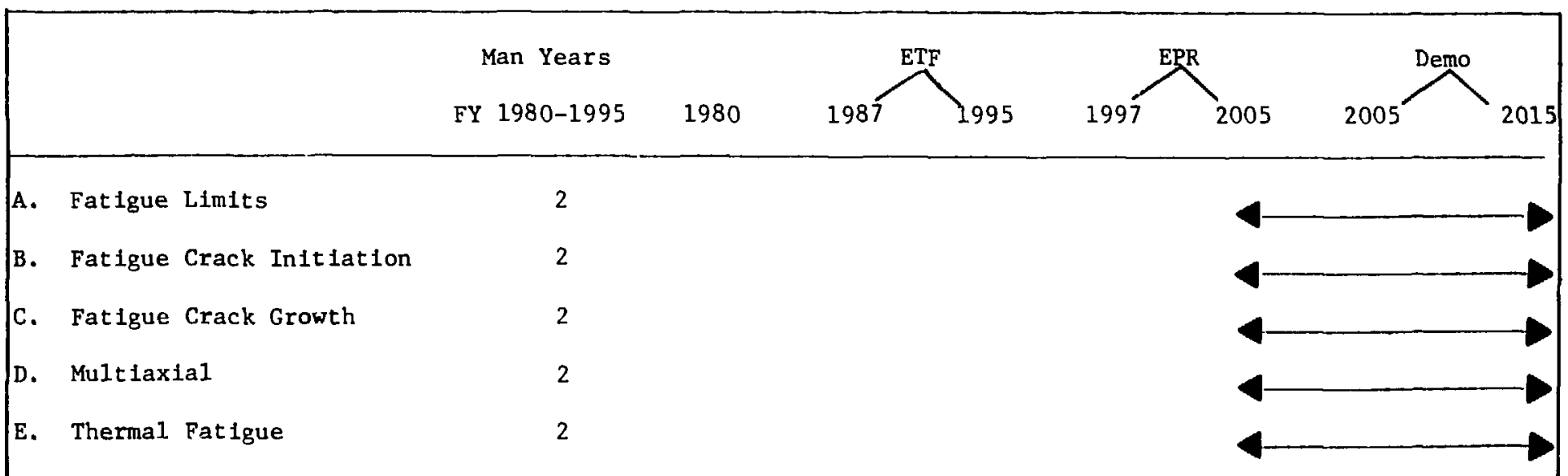


Table 3-8.

Material: Ferritic Steels and Austenitic Steels

Environment: Air or Inert Gas

Objective: To evaluate mechanical propertles design data for ETF and EPR.

Scope: The tensile, load relaxation, creep, creep-to-rupture and fracture toughness properties will be examined.

\begin{tabular}{|c|c|c|c|c|c|c|c|}
\hline & \multicolumn{2}{|l|}{ Man Years } & ETF & \multicolumn{2}{|c|}{ EPR } & \multicolumn{2}{|c|}{$\overbrace{\text { Demo }}^{\text {Den }}$} \\
\hline & FY $1980-1995$ & 1980 & $1987 \quad 1995$ & 1997 & 2005 & 2.005 & 2015 \\
\hline A. Tensile Properties & 2 & & & & & & \\
\hline $\begin{array}{l}\text { B. Creep and Load Relaxation } \\
\text { Properties }\end{array}$ & 3 & & & & & & \\
\hline $\begin{array}{l}\text { Creep-to-Rupture } \\
\text { Properties }\end{array}$ & 3 & & & & & & \\
\hline p. Fracture Toughness Properties & 6 & & & & & & \\
\hline
\end{tabular}


1. P. Patriarca, S. D. Harkness, J. M. Duke, and L. R. Cooper, Nuclear Technology, 28, (1976) 516.

2. The Fusion Reactor Materials Program Plan, DOE/ET-0032 (July 1978).

3. I. 0. Bohachevsky, L. A. Booth, T. G. Trank, and J. H. Pendergrass, "Loads and Stresses in ICF Reactors," LAUR-79-432, 5th Int1. Conf. on Structural Mechanics in Reactor Technology, Berlin, Germany, (August 1979).

4. I. 0. Bohachevsky, "Scaling of Reactor Cavity Wa11 Loads and Stresses," LA-7014-MS (Nov. 1977).

5. L. A. Glenn and D. A. Young, "Dynamic Loading of the Structural Wall in a Lithium Fall Fusion Reactor," Lawrence Livermore Laboratory, UCRL-82125 (1978).

6. J. Weertman, "Potential Fatigue Problems in First Wall Laser-Controlled Fusion Reactors," Los Alamos Scientific Laboratory, LA-5664-MS (1974).

7. L. A. Booth (Compiler), "Central Station Power Generation by LaserDriven Fusion," Los Alamos Scientific Laboratory, LA-4858-MS, Vol. 1 (February 1972).

8. S. Majumdar, B. Misra and S. D. Harkness, Fusion Reactor Design Concepts, IAEA-TC-145/35, Vienna, Austria (1978) 549-560.

9. P. S. Maiya, "Considerations of Crack Initiation and Crack Propagation in Low-cycle Fatigue," Scripta Metallurgica, Vo1. 9, (1975) 1141-1146.

10. P. S. Maiya, "Effects of Surface Roughness and Strain Range on the Lowcycle Fatigue Behavior of Type 304 Stainless Stee1," Vol. 9, (1975) 1277-1282; also Mater. Sci. Eng. 21, (1975) 57-62.

11. E. Siebel and M. Gaier, "The Influence of Surface Ruughness on the Fatigue Strength of Steels and Non-Ferrrus Alloys," The Engineers' Digest, (1957) 109-112.

12. E. Glenny and T. A. Taylor, "A Study of the Thermal-Fatigue Behavior of Metals," Inst. Metals, 88, (1959-1960) 449.

13. Proceedings of a Workshop on "Time Dependent Fracture of Materlals at Elevated Temperatures", CONF-790236, Div. of Materials Sciences, DOE (1979). 


\section{LITHIUM COMPATABILITY AND OTHER ENVIRONMENTAL EFFECTS}

\subsection{Scope}

The utilization of liquid lithium in several ICFR design concepts is proposed for first wall protection. ${ }^{1}$ In these design concepts liquid lithium is also used for tritium breeding and as the primary coolant. The compatibility of liquid lithium with various first wall, blanket and optical systems materials is therefore an important consideration for ICFR development.

As mentioned earlier, the choice of the structural materials for near term ICFR systems can be confined to the ferritic steels and austenitic stainless steels. Of these two types of material, more information on lithium compatibility is available for austenitic stainless steels. These data suggest potential problems in this area. For long term applications, the operating temperature for an austenitic stainless steel-1ithium system appears to be restricted to below $500^{\circ} \mathrm{C}$. Properties of ferritic steels may not be significantly superior to those of austenitic stainless steels. Since the former steels are strong candidates for structural materials, it is essential that efforts are made to qualify these steels for lithium compatibility and to accumulate sufficient information to specify operating conditions of near term ICFRs.

An important finding of the reported work is that ferrous alloys will suffer intergranular corrosion and penetration and that the rate of attack depends on the stress level, the composition of the lithium, and the composition and structure of the material. 2,3,4 These observations suggest that the fracture and fatigue properties in lithium can be significantly different from those obtained during post-exposure testing, and in-situ experiments are needed. In this section, therefore, facility requirements for mechanical testing in lithium will be considered as well as the development of lithium handling capabilities.

There are design concepts for which liquid lithium will not be used.1 other environmental effects will exist and will be considered in this section. 


\subsection{Background}

It is generally recognized that lithium is more corrosive tcward ferrous alloys than sodium, $2,3,4$ and experience and data gained in the liquid metal fast breeder reactor program will therefore be of limited applicability. The various modes of lithium attack that have been identified include:

1) Simple solution

2) Intergranular penetration

3) Impurity reactions

4) Temperature gradient mass transfer

5) Concentration gradient mass transfer or dissimilar metal mass transfer.

The following variables have been found to influence the rate of attack in ferrous alloys:

1) Temperature

2) Temperature gradient

3) Cyclic temperature fluctuation

4) Purity of liquid lithium

5) Flow velocity

6) Number of materials in contact with the same liquid lithium

7) Composition and structure of the material

8) Stress state.

The attack by intergranular penetration is a phenomenon which requires special attention. This type of attack does not occur in the sodium environment and hence is not a problem in sodium technology. One can easily visualize that the grain boundary process involved will enhance crack initiation and propagation under an applied load, and lead to severe degradation of the mechanical strength of the materlal. A thorough investigation of this phenomenon is required to obtain Information to be used for materials selection and reactor design.

Based on reported work, 2,3,4 intergranular attack is caused by the fact that the free energy of formation of lithium carbide and nitride 1s lower 
than that of some of the chromium carbides and nitrides. The difference in the free energy of formation provides the driving force for lithium to diffuse into the steel and to reduce chromium carbides and nitrides. It is well known, especially in ferritic steels, that different carbides of varied stability exist depending on the prior heat treatment and thermal aging conditions. 5 The rates of intergranular attack in weld metals and in the heat affected zone can therefore be expected to differ from that in the base metal. The rate of attack is also likely to change with time during service due to thermal aging. The carbon and nitrogen content of the steels and the composition of major components should have a strong effect on the resistance to intergranular attack. Recently, irradiation-induced segregation and precipitation have been shown to occur. ${ }^{6}$ These processes could potentially form carbides of varied stability and thus have an indirect effect on the rate of attack.

A significant finding of the past work on intergranular lithium attack is that the rate of attack increases with increasing carbon and nitrogen content in lithiun. This effect may be caused by the carbon and nitrogen diffusing along grain boundaries and forming chromium carbides and nitrides which are subsequently reduced by the 1 ithium. The observed dependence suggests the importance of controlling the impurity level in lithium.

Based on free energy considerations, group IV, V, and VI elements in the periodic table can cause chemical effects similar to those produced by carbon and nitrogen described above. Pellet debris will contain elements of these groups and will enter lithium as impurities. In addition to the possibility of enhancing intergranular attack, some of these elements (e.g., $\mathrm{s}$, $\mathrm{Sb}, \mathrm{P}$, etc.) may enter grain boundaries and produce embrittlement effects similar to those resulting from temper embrittlement. In the proposed program, these possibilities will be investigated so that potential problem areas can be identified and near term remedies sought if required.

The effects of stress on intergranular attack have been reported by several investigators. ${ }^{2,3}$ Under loading conditions where intergranular crack initiation and propogation occur, it will be difficult to separate stressassisted intergranular attack from environment-enhanced crack initiation and growth, and in-lithium mechanical testing becomes important. 
A part of lithium technology needed for the materials program is the capability of impurity removal and composition control. ${ }^{7,8}$ The techniques for continuous monitoring of the impurity level in the lithium loop are also required, since the importance of performing materials testing in well-controlled and characterized liquid lithium is recognized. It is expected that the development of the experimental capabilities for the proposed program will also serve the purpose of establishing the required lithium technology in the areas mentioned above.

Hydrogen is known to cause embrittlement at lower temperatures, to form hydrides in some metals, e.g., refractory alloys. It is also known to react with carbon in steel to form methane bubbles at grain boundaries. The latter reaction may be enhanced by irradiation directly and indirectly by irradiationinduced precipitation and segregation, which may form carbides of lower stability. Since similar problems will also occur in magnetic fusion materials, the investigation of hydrogen effects other than its intluence on fatigue properties will not be included in the proposed program.

Mirror materials will be in contact with liquid lithium and lithium vapor and also with pellet debris. In the SOLASE concept ${ }^{9}$ liquid lithium is not used, but chemical reactions between graphite and unburned fuel and pellet debris may occur. The energy sink material, used in reactor concepts where the first wall is protected magnetically, will interact with charged particles and pellet debris. These are potential problem areas where materials development is required and will be included in the proposed program.

\subsection{Data Requirements}

\subsubsection{Lithium Compatibility}

Engineering data are required on the rate of various modes of lithium attack and their dependence on the variables given at the beginning of the section on background. Mechanistic studies are required to provide a basis for data correlation and extrapolation and for materials selection. In the following, the different modes of lithium attack will be discussed separately. 


\subsubsection{Solution Attack}

The rate of solution attack can be measured by weight loss and dimensional changes. The rate of solution of the major elements in ferrous alloys is known to vary from one element to the other. 2 The characterization of the depth and the composition and structure of the affected surface region in contact with liquid lithium is also a necessary part of data collection, since the preferential removal of nickel has been shown to produce a ferrite surface layer in austenitic stainless steels. The experiment should be performed as a function of temperature, temperature cycle, flow rate and controlled concentration of non-metallic elements and other impurities in 1iquid 1ithium. Some measurements should be performed with irradiated specimens whose surface condition has been controlled during irradiation.

The solubilities of the major elements in ferrous alloys are important fundamental parameters that define the driving force for solution attack. Literature values should be confirmed under controlled experimental conditions and further tests conducted to determine the effects of impurities which are expected in an ICFR environment.

\subsubsection{Intergranular Penetration}

The possible mechanisms involved in intergranular lithium attack have been discussed in the background section. This is an area where extensive effort is expected. Controlled experiments are required to determine the rate of attack as a function of temperature, temperature cycle, flow rate, concentration of non-metallic elcments and other impurities in lithium, and the stress state. The variations in composition and microstructure in the steels resulting from fabrication histroy, heat treatment, thermal aging and irradiation as discussed previously are expected to have a strong effect on the rate of attack. A systematic investigation of the influence of these materials variables should be made.

The rate of attack can be measured from the depth of penetration. The characterization of the corrosion product in the gratn boundary and the related compositional and microstructural changes in the grain matrix are of major importance. Various advanced diagnostic tools are avallable today and should be used in the proposed program. 
The effect of stress has been shown to be an important consideration. Only the in-1ithium mechanical testing under static loading is considered in this section, since the in-lithium mechanical testing under cyclic loading is included in the section of the proposed program dealing with fatigue. It is expected that these experiments will yield information on the effects of stress on the rate of intergranular attack as well as data on the effect of lithium environment on creep, stress to rupture, and fatigue properties of the proposed structural materials.

\subsubsection{Impurity Reactions}

It is well established that interstitial elements such as carbon and nitrogen can enter or leave a ferrous alloy depending on the chemical potential of the particular element in lithium and in the metal. The resulting compositional and microstructural changes in the material can strongly influence its mechanical properties; for example, severe decarburization can significantly reduce the creep strength of the material. The rate of the reaction can be determined from the depth of the affected zone and from the resulting compositional and microstructural changes. Since long term in-lithium mechanical testing can be costly, post-exposure mechanical testing will be utilized whenever possible in the proposed program. Post-exposure testing can be justified for cases where mechanical properties are governed by the bulk properties and where the anticipated compositional and structural changes can be produced by proper lithium exposure treatment.

An important consideration for the impurity reactions is to predict the rate of reaction at long times, so that one can accurately calculate, for example, the depth of the decarburized zone and estimate the remaining mechanical strength. For this purpose it is essential that the thermodynamics data that govern the driving force for impurity reactions be well established. Experimental data are also needed on the kinetics constants such as the diffusion coefficient or the reaction rate constants for the rate determining processes. If literature data are not available, efforts are expected to be made to determine the values of these basic parameters.

Carbon and nitrogen in lithium are known to enhance the rate of intergranular attack. Other impurities, as discussed in the section on background, such as $\mathrm{Pb}, \mathrm{Sb}$, etc. may cause similar effects. These impurities may 
also enter the grain boundaries and cause embrittlement. In addition to determining the rate of reactions of the latter impurities, it is important that the relevant thermodynamic and kinetic data be established for data correlation and extrapolation.

\subsubsection{Temperature Gradient and Concentration Gradient Mass Transfer.}

Temperature gradient mass transfer is known to occur in lithium loops. ${ }^{2}$ Concentration gradient mass transfer can occur between dissimilar materials and between weld metal and base metal. It is possible that temperature gradients and concentration gradients will co-exist, depending on reactor design. The mass transfer rate should be measured systematically as a function of temperature, temperature gradient, composition gradient, temperature cycle, flow rate, and the purity of liquid lithium. The rate can be determined from weight and dimensional changes. It is important, as for other modes of attack, that the surface region of the material be well characterized in terms of the depth of the affected zone, composition, and microstructure.

Rate equations based on thermodynamics and kinetics as well as on fluid dynamics considerations should be established from experimental data. Since the temperature and concentration gradients in a reactor can vary over a large range, the rate equations obtained will be useful for reactor design.

\subsubsection{Materials Variables and Experimental Conditions}

As pointed out previously, the initial phase of the materials program will emphasize ferritic steels with austenitic stainless steels as the alternative structural material. Several ferritic steels ${ }^{10}$ of varied chromium content are strong candidates and will be included in the proposed program. It is recognized that the properties of ferritic stainless steels are sensitive to heat treatment, fabrication histroy, thermal aging, and minor alloy additions such as carbide stabilizers. The effects of these materials variables will be investigated systematically. Consideration of weldments and the heat-affected zone will be included with the materials variables. 
The various experimental conditions of interest can be summarized as follows :

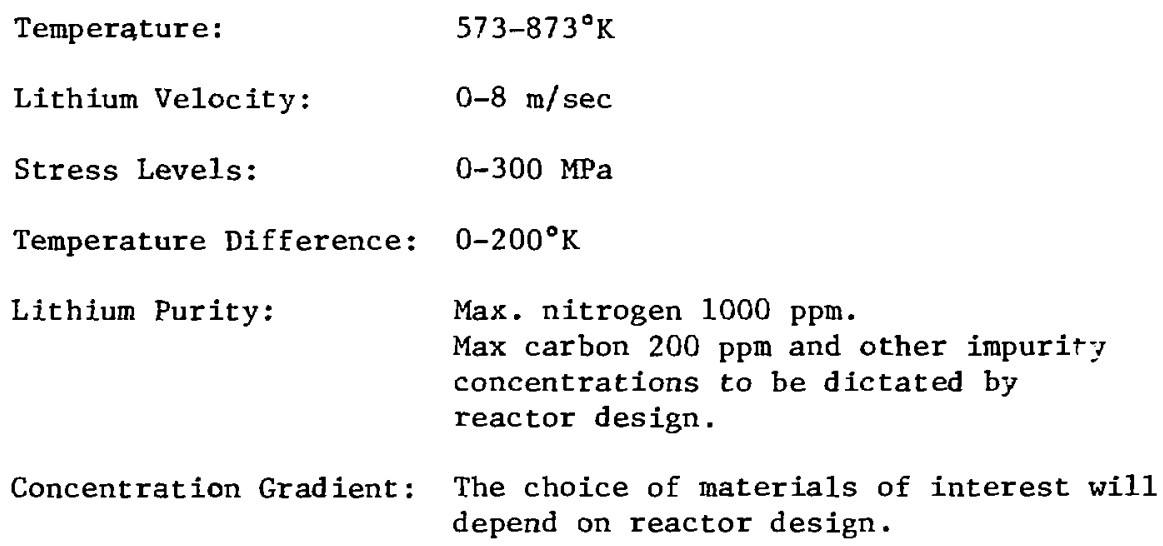

Concentration Gradient: The choice of materials of interest will depend on reactor design.

\subsubsection{Other Environmental Effects}

Exploratory work to investigate the effects of ICFR environment on mirror and energy sink materials should be initiated. If problem areas exist, they may be solved in part by materials selection rather than by materials development. The interaction between graphite and unburned fuel and pellet debris can be simulated by ion bombardment and other techniques. Some of the same interactions will also occur in magnetic fusion reactors and the proposed program will utilize results of materials research designed for magnelir. fusion reactor development.

\subsection{Strategy}

The 1ithium loop used in the test programs should have controlled nitiogen, carbon, oxygen, and hydrogen levels as well as other impurity concentration levels. The capabllity for continuous monitoring of these compositions is required.

Lithium loop tests need to be conducted with progressive complexity of experimental conditions so that the effects of a particular variable can be isolated. For test programs prior to EPR, 1ithium loops of sma11 scale will be used. After EPR, some of these small loops can be used for long time testing. It is planned that large scale 1ithium loops will be constructed and be avallable after EPR. The large scale loors could actually be a part of 
EPR. These large loops can be used to test the synergistic effects of several critical variables, to confirm the validity of the various rate equations developed for reactor design, and to proof-test the various impurity trapping and removal capabilities as well as the equipment for monitoring the impurity level.

Mechanical testing in-1ithium is emphasized in the proposed program because of the influence of stress on corrosion. Post-exposure testing will be utilized to determine the effects of impurity reactions such as decarburization on the mechanical properties. Compositional and microstructural changes produced by long term lithium exposure with andowithout radiation will also be investigated.

An important requirement of lithium research is developing rate equations for lithium attack that are capable of predicting the long-time behavior of the material. For this purpose, mechanistic knowledge of various modes of attack is essential. The various advanced diagnostic tools available today should be fully utilized to characterize the composition and structure of corrosion products and the related compositional and microstructural changes in the material. The results of detailed chemical and physical analyses should aid considerably in understanding the mechanisms involved in lithium attack. 
Table 4-1.

Material: Ferritic Steels and Austenitic Stainless Steels

Environment: Flowing Lithium (Small scale loops)

Objective: To evaluate lithium corrosion and mass-transfer in lithium.

Scope: The rate of various modes of lithium attack will be measured as a function of impurity levels, environmental and materials parameters, and rate equations for design applications will be developed.

A. Corrosion and Mass Transfer
(a) Lithium loop construction
and development
(b) Temperature and temperature
(c) Temperate
(d) Fradient
(e) Purity of rot lithium
(f) Materials variables,


Table 4-2.

Material: Ferritic Steels and Austenitic Stainless Steels

Environment: Flowing Lithium (Small scale loops)

Objective: To evaluate effects of lithium on mechanical properties.

Scope: The effects of stress state on corrosion rates and the effects of a 1 ithium environment on mechanical properties (fatigue, etc.) of steels will be investigated.

Effects on Lithium on
Mechanical Properties
(a) Lithium loop construction
and development
(b) Temperature and temperature
cycle
(d) Purity of lithium
(e) Materlals variables,
composition and structure
(f) Stress state (Fatigue, Creep)
- In 1ithium mechanical testing


Table 4-3.

Material: $\quad$ Ferritic Steels and Austenitic Stainless Steels

Environment: Flowing Lithium

objective: To evaluate lithium compatibility design data for demonstration plant and beyond.

Scope: The rate of various modes of lithium attack will be measured as a function of environmental - and materials parameters and rate equations for design applications will be developed.

$$
\text { Maq Years }
$$

FY 1980-1995

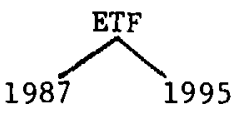

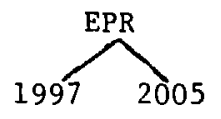

A. Loop construction and development

B. Lithium attack measurements

(a) Solution attack

(b) Intergranular attack

(c) Impurity reactions

(d) Temperature and concentrution gradient mass transfer 


\section{REFERENCES}

1. J. A. Maniscalco, D. H. Berwald and W. R. Meier, "The Materlals Implications of Design and Systems Studies for Inertial Confinement Fusion Systems", First Topical Meeting on Fusion Reactor Materials, Miami Beach, FL., (Jan. 1979) to be published.

2. J. H. DeVan, J. E. Stelle and A. E. Morris, "Review of Lithium IronBase Alloy Corrosion Studies", ORNL/TM-4927, Oak Ridge National Laboratory (1976).

3. D. L. Olson and W. L. Bradley, Proc. of International Conf. on Liquid Metal Technology in Energy Production, ANS-ATME Conf. Proc. No. 760503-P1, Champion, Pa., (1976) 446.

4. N. J. Hoffman, private communication.

5. R. G. Baker and J. Nutting, JISI, 192, (1959) 257.

6. P. Wilkes, "Phase Stability Under Irradiation--A Review of Theory and Experiment", University of Wisconsin, UWFDM-271 (Nov. 1978).

7. J. R. Weston, W. F. Calaway, R. M. Yonco, J. B. Hines and V. A. Maroni, "Control of Impurities in Forced Circulation Lithium Loop Systems", presented at "Corrosion 79" (March 12-16, 1979) to appear in Corrosion.

8. J. R. Weston, W. F. Calaway, R. M. Yonco, E. Veleckis and V. A. Maroni, "Experimental Studies of Processing Condltions for Liquid Lithium and Solid Lithium Alloy Fusion Blankets", to appear in Proc. of Third ANS Topical Meeting on Technology of Controlled Nuclear Fusion, Santa Fe, New Mexico (May 9-11, 1978).

9. SOLASE--A Laser Fusion Reactor Study, University of Wisconsin, UWFDM-220.

10. P. Patriarca, S. D. Harkness, J. M. Duke and L. R. Cooper, Nuclear Technology, 28, (1976) 516.

11. K. Natesan, T. F. Kassner and Che-Yu Li, Nuclear Technology, 15, (1972) 224. 


\section{EXECUTIVE SUMMARY}

The requirements of the inertial confinement fusion program generate issues of two types when materials development is considered. Technical questions arise because of unfamiliar environmental conditions such as pulsed irradiation, cyclic mechanical and thermal loadings, and extensive use of liquid lithium. Other issues arise because of the time frame for engineering development. In this plan, we attempt to balance both technical and scheduling concerns.

The engineering development schedule, shown in Fig. 5-1, calls for design decisions on the Engineering Test Facility (ETF) in 1987. Because of the nearness of this date, it is unlikely that any alloy system not already well characterized will be used in the construction of the ETF. The Experimental Power Reactor (EPR) will be the first large volume, high plant factor facility capable of generating materials data required for engineering design. Thus, the schedule for development of inertial confinement fusion suggests an approach to materials development that divides the time frame into two periods.

During the first time period, which will extend from the present until the date of initial EPR operation or about the year 2000, the development effort should concentrate on the qualifications of the ferritic stainless steels as reference structural materials and the austenitic stainless steels as alternates, with only a low level of activity on other alloy systems. The second time period extends from the date of initial EPR operation through the construction of the first commercial machine. During this period, large scale testing of other systems along with those previously selected should occur using the EPR itself as a major test bed. The justification for this strategy is based, in part, on the promise and potential of the ferritic stainless steels for fusion application. This class of commerclally available steels appears to be resistant to void swelling and helium embrittlement, while being compatible with liquid lithium. Key questions about its applicability do remain, however, and must be addressed at an early stage in the program. One of the areas of concern is the compatibility of pellet impurities with the ferritic and austenitic stainless steels.

The first decision point in the program occurs in 1985, when a decision as to whether or not the ferritic stainlessess are appropriate for the construction of the ETF and remain promising for EPR should be made (see Fig. 5-2). 


\section{Fusion Development}

(Reference Budget Case)

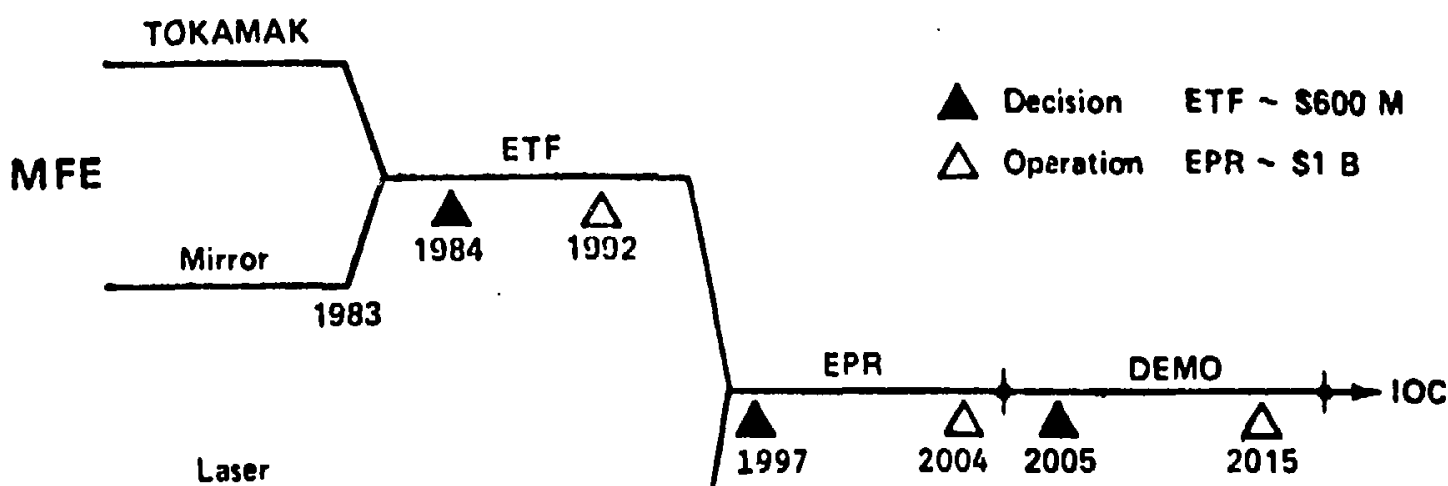

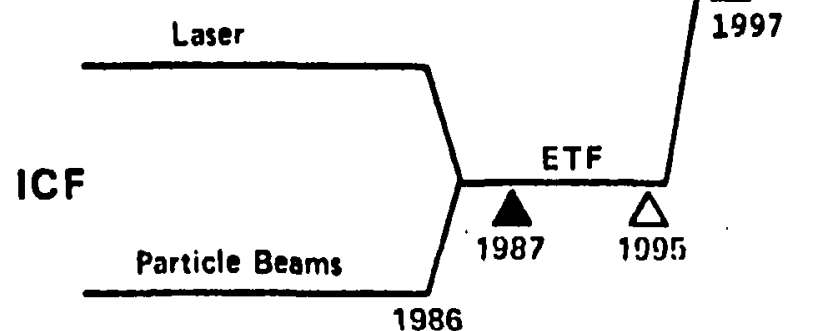

Key Milestones

Cumulative Costs To Key Milestones

ETF

ETF Decision Operation
20042005

Time vs Dollar Tradeoff

- Post-EPR Decision Program Could Be Accelerated BY Dollar Rampup with Greater Certainiy

- Issue Today is Program Through ETF Period Up To EPA Decision Point

S4 B S11 B S18B

78.8556-M/12.20

Fig 5-1. Schedule for fuston reactor development [Ref. J. M. Deutch, Department of Energy Policy for Fusion Energy, DOE/ER-0018, Sept. 1978.] 
If they are not appropriate, then the following period can be spent in an intensified effort to qualify an austenitic stainless steel for ETF application. Otherwise, the two years prior to the ETF decision date will be used to select a specific ferritic alloy from the range of possibilities. It should be noted that much experience can be gained if the same material can be used for both the ETF and the EPR. Thus, if it becomes clear that the ferritics cannot be used at the higher dpa levels required by EPR, they should probably not be used in ETF.

A second major question in the materials development program is whether or not the waterfall concept can be engineered for the driver system most appropriate for commercial development. The incentive for developing the waterfall system is based on its amelioration of radiation aamage and pressure pulse effects with the consequent extension of the structure lifetime. If the concept is not workable, the severity of the first wall environment increases, and the focus of the materials program may change. Thus, a two-pronged effort that closely couples chamber design activities with the material development effort is also suggested. This effort will then support the decisions on vessel design that must be made for ETF in about 1990 and for the EPR in about 1999.

If it can be shown that pulse effects are not significant, and that available alloys such as the ferritic stainless steels are capable of surviving neutron wall loadings of 40 or more $M-y r / m^{2}$, then the additional englneering complexities of the waterfall approach may not be justified. A decision on the feasibility of the concept has been scheduled in the material development plan for 1985. If the waterfall design is proven, then decisions on its use in ETF and EPR blankets are indicated for 1988 and 1998.

A third major decision for the program is whether or not a dedicated materials test facility is needed to allow the confident construction of an EPR. This decision depends strongly on the nature of pulsed neutron damage and its effects on the microstructure and mechanical properties of materials. It is a conclusion of this assessment that currently available or planned accelerators and fission reactors can be used to assess the importance of pulsed effects on microstructural development and to determine their relative magnitude. Clearly if the effects are large, there will be a strong incentive to develop and operate a separate materlals test facllity. If not, then 
1. Are Ferritic Stainless Steels Applicable?

High dose effects

High He effects

DBTT shift - Tougheners

Weld performance

High cycle fatigue

2. Are there Certain Pellet Impurities that are Incompatible with Using a Ferritic or Austenitic Structure?

3. Can a Waterfall Concept be Used?

(a) ETF Blanket design picked

(b) EPR Blanket design picked

4. Is a Dedicated Facility Needed to Build EPR?

5. What are the Magnitude of Pulsed Effects on Microstructure Development?

6. What are the Costs of a Low Gain (225) H1gh Duty Factor Material Test Facility?

7. Define Severity of CorrosionFatigue Problem in Li.

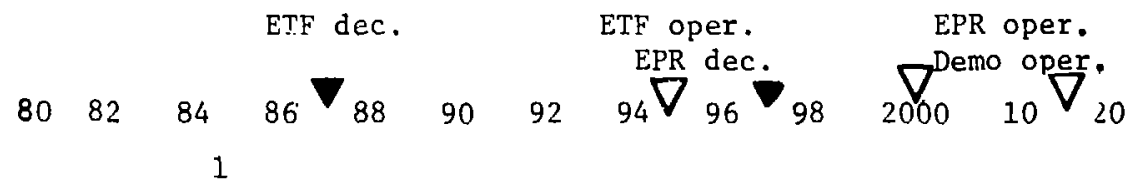

2

$3 a$

$3 b$

Figure 5-2. Key questions and decision rates in the Material Development Program for an inertial confinement fusion reactor. 
it is felt that the EPR itself can serve as the central high volume test facility to qualify materials for the demonstration plant and the first commercial machines. The large scale development of more advanced alloy systems, such as those based on vanadium, should be delayed until the applicability of the ferritic stainless steels has been determined, and the EPR is available for use as an irradiation test bed.

As input to the decision on a materials test facilfty, an estimate of the costs and schedule for building a high duty factor, low gain materials test facility must be available by the 1986 decision date.

All of the inertial confinement designs will be subject to high cycle fatigue loading. It is important that testing of this key property area be conducted on candidate materials for the construction of the EPR. It will be particularly important to determine the effect of surface damage processes on the high cycle fatigue life-time.

The discussion above is summarized in Fig. 5-2, which 1 ists key questions that the materials development program must address and gives dates when information should be available so that timely design decisions can be made. Answers to questions $1,2,5$, and 7 depend directly on information that will be obtained from the technical programs discussed in this report; major tasks for these programs have been listed in Tables 5-1 to 5-4 along with schedules for their completion. 
Table 5-1.

Question: Are ferritic stainless steels applicable for inertial confinement fusion reactors?

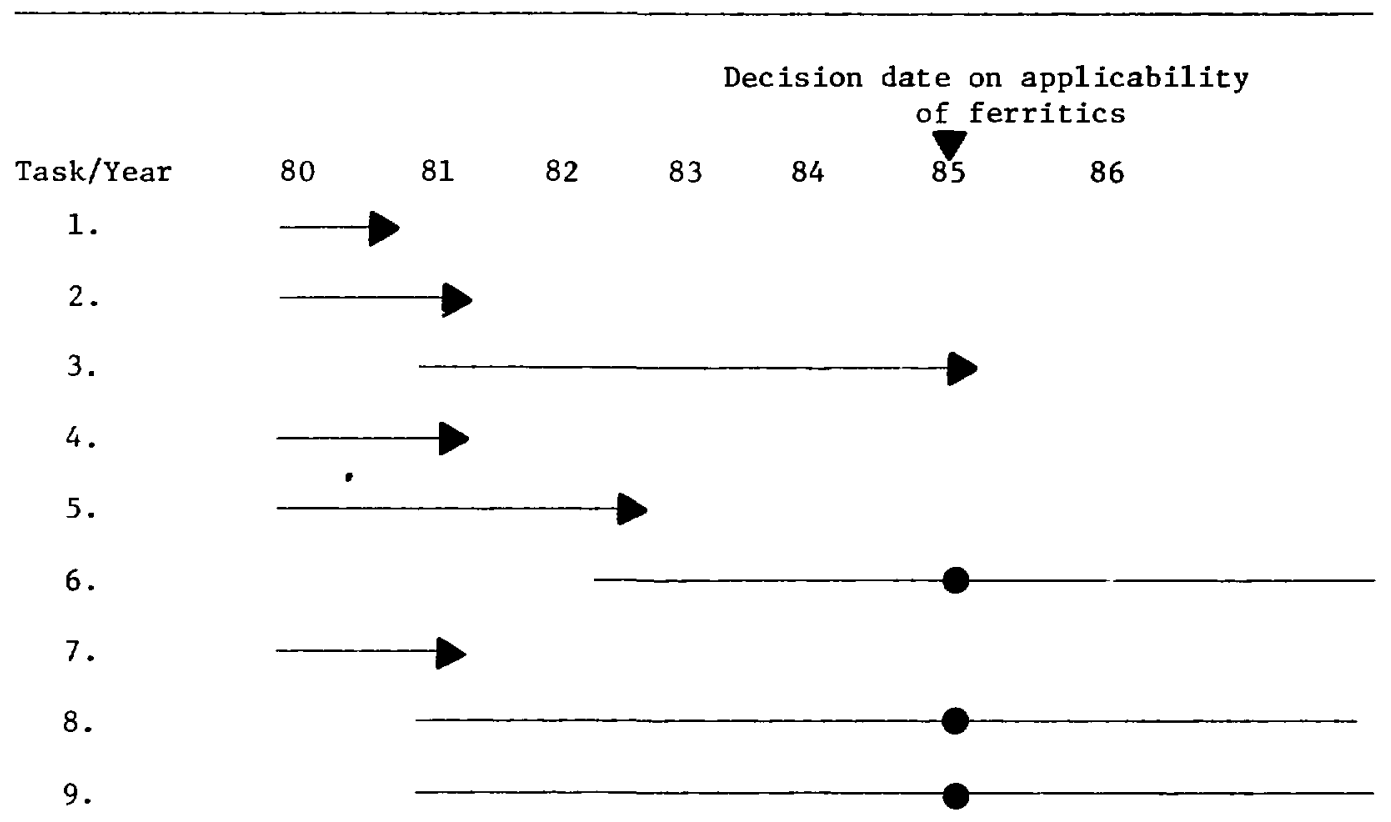

Interim assessment report.

1. Select candidate ferritic alloys.

2. Plan test matrix for determining effects of irradiation, Li soaking, thermal aging on toughness (including DBTT) on both base and welded samples of different initial heat treatments.

3. Define effects of ICF environment on toughness sufficiently to allow construction decision on ETF.

4. Define welding practice for candidate ferritic steels.

5. Develop plan for determining the relationship between mechanical properties and microstructure as a function of dose up to $400 \mathrm{dpa}$ using tnput from study on pulse effects.

6. Conduct irradiation program using fission reactors and accelerators to define mechantcal properties as a function of dose.

7. Develop plan for defining the role of helium in the ferritic stainless steels. 
Table 5-1. (Continued)

8. Test for resistance to helium embrittlement using cyclotron injected and nickel-containing neutron irradiated alloys.

9. Conduct high cycle fatigue testing including corrosion fatigue effects. 
Table 5-2.

Question: Are there certain pellet impurities that are incompatible with using a ferritic or austenitic structure?

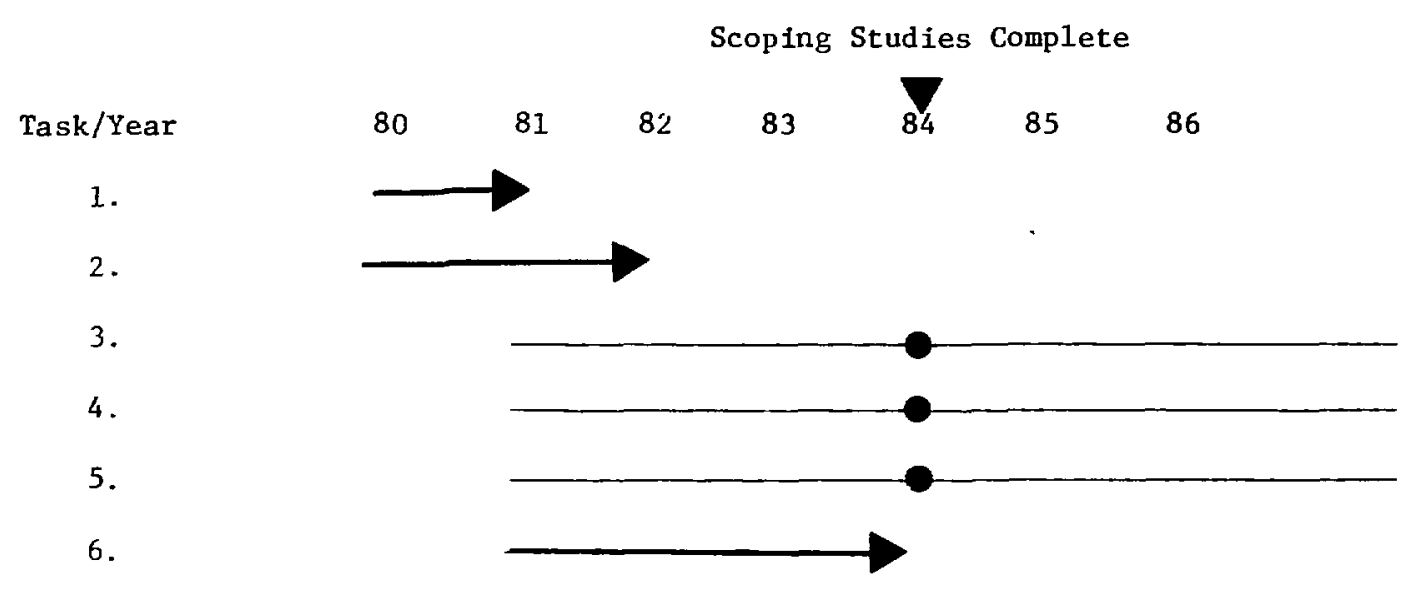

1. Select materials for experiments.

2. Plan test matrix to study effects of pellet impurities on lithium compatibility.

3. Perform experiments in lithium on the rate of solution attack, intergranular attack, impurity reactions and temperature gradient mass transfer with controlled impurity levels.

4. Perform mechanical testing to evaluate post exposure fracture toughness and fatigue properties.

5. Develop rate equations for 1 ithium attack under the influence of pellet impurities.

6. Analyze results and produce preliminary recommendations. 
Table 5-3.

Question: What are the magnitudes of pulsed effects on microstructural deveiopment?

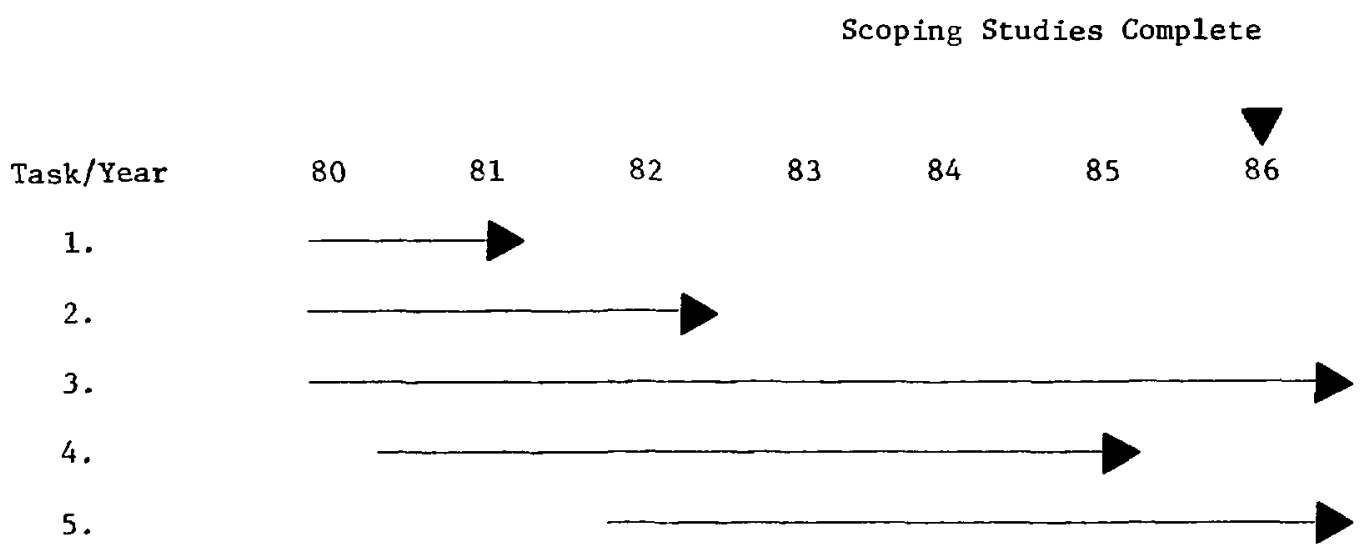

1. Select materials for experiments.

2. Plan test matrix for study of damage rate and pulse duration effects on microstructural components.

3. Perform theoretical calculations with available models to predict magnitude of effects and analyze experimental results.

4. Conduct irradiation experiments in accelerators and fission reactors.

5. Analyze results. 
Table 5-4.

Question: Define severity of corrosion fatigue problem in lithium.

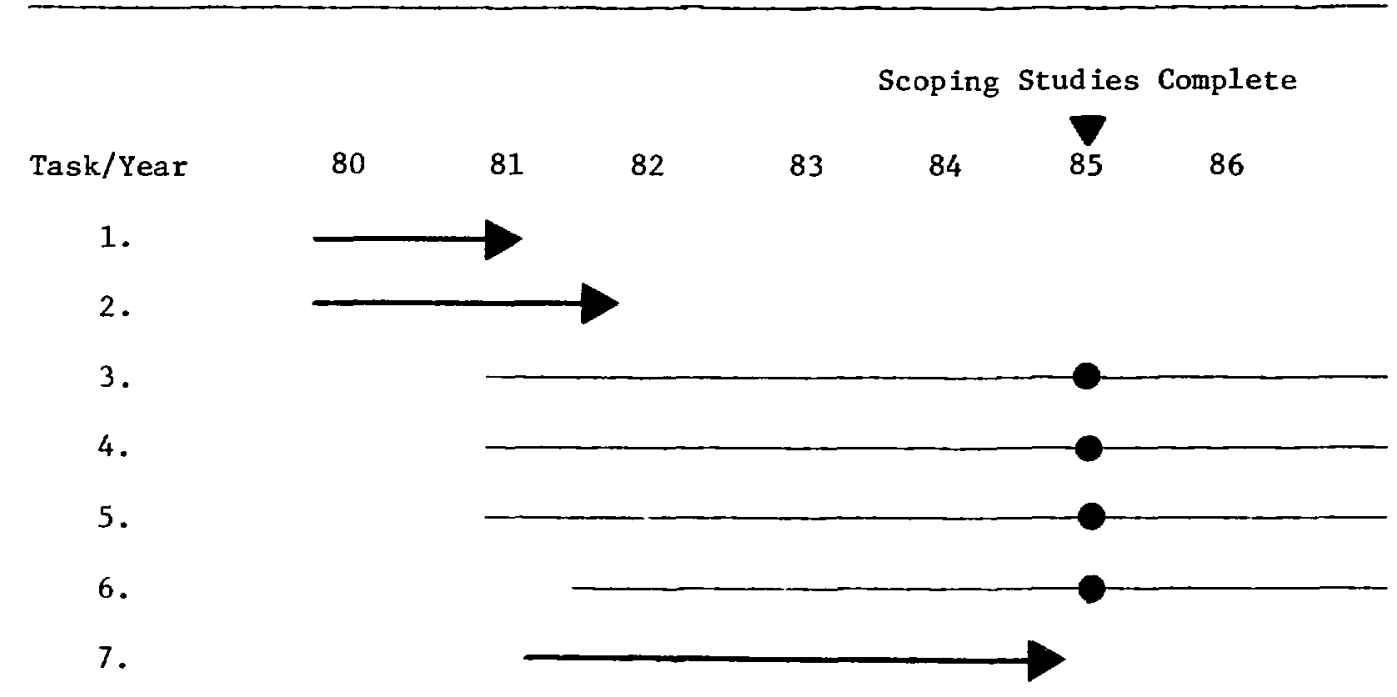

1. Select materials for experiments.

2. Plan test matrix to evaluate corrosion fatigue properties of ferritic steels and austenitic stainless steels in 1ithium.

3. Perform experiments in lithium on the rate of solution attack, intergranular penetration, impurity reactions and temperature gradient mass transfer under controlled conditions.

4. Perform fatigue testing in lithium.

5. Perform post-exposure fatigue testing.

6. Develop rate equations for lithium attack.

7. Aitalyze results and produce preliminary recommendations. 